\title{
amélioration des sols par inclusions rigides verticales application à l'édification de remblais sur sols médiocres
}

\author{
amelioration of soils by vertical rigid piles \\ application to construction of embankments on soft soils
}

O. COMBARIEU

Laboratoire régional des Ponts-et-Chaussées de Rouen *

Rev. Franç. Géotech. n` 44, p.p. $57-79$ (juillet 1988)

\section{Résumé}

Ce rapport examine les conditions de mise en œuvre d'un réseau d'inclusions verticales rigides, destiné à améliorer un sol compressible chargé par un remblai d'apport. II est développé une méthode de calcul de dimensionnement du réseau qui est confrontée aux données ou méthodes expérimentales existantes.

\footnotetext{
Abstract

This report looks into the state of use of vertical rigid piles network, to improve a compressible soil under fill. A design method of this network is developed, which is compared with existing experimental results or methods.
} 


\section{INTRODUCTION}

L'éventail des méthodes d'amélioration des sols de fondations est, à l'heure actuelle, largement diversifié, depuis les anciens procédés de consolidation des sols mous par pilotis, jusqu'aux techniques les plus modernes, telles que pilonnage intensif ou congélation, entre autres.

Ces techniques modernes, de développement récent, ont fait l'objet d'une publication (QUEYROI et al., 1985) sous forme d'une note d'information technique, précisant les procédés, leurs performances et leurs limites d'emploi ; cette note a fait largement appel à l'expérience de ses auteurs, et aux expérimentations spécifiques auxquelles le réseau des Laboratoires des Ponts et Chaussées a été appelé à connaître ou à participer.

Un procédé ancien, très largement millénaire, et évoqué ci-dessus est la consolidation par « pilotis ». Cette technique beaucoup utilisée autrefois, aux justifications empiriques résultant de la constatation qu'elle s'avérait efficace, puis oubliée, du moins dans les pays les plus développés, fait actuellement une réapparition timide. Quelques rares publications récentes font état de son utilisation avec encore, pour la plupart, une justification technique empirique mais économiquement fondée, ou au contraire pour d'autres avec une justification étayée par des moyens de calcul sophistiqués, tels les éléments finis.

L'étude qui suit a pour but d'examiner les aspects sous lesquels on peut envisager d'améliorer un sol par inclusions rigides et quels problèmes on peut imaginer de résoudre par un tel procédé. On propose, pour une certaine configuration, une méthode de calcul relativement simple, qui est confrontée à des applications réelles puisées dans les quelques données de la littérature technique.

\section{RÉFLEXIONS SUR L'AMÉLIORATION PAR INCLUSIONS VERTICALES RIGIDES}

Plus généralement, l'amélioration par inclusions rigides s'étend aux inclusions inclinées, dont l'essor a vu le jour en Italie (pali-radice, ou pieux racines). On se limite volontairement ici aux éléments verticaux, à l'évidence, plus simples à justifier.

Le renforcement consiste en un réseau d'inclusions, de maillage donné et intéressant tout ou fraction de l'épaisseur des sols médiocres. On peut d'ailleurs ramener la schématisation des sols à un simple bicouche, comportant un sol de mauvaise qualité surmontant un sol résistant, ce dernier n'ayant nullement besoin d'être amélioré. Ce schéma simple conserve toute sa généralité aux réflexions qui suivent.

Le sol médiocre d'épaisseur H (fig. 1) est donc « cloué * par un réseau d'inclusions rigides, dont la longueur, a priori, peut être inférieure à $\mathrm{H}$; ce réseau est caractérisé par le diamètre $2 \mathrm{R}$ des inclusions, et l'espacement moyen $2 \mathrm{~b}$, paramètres géométriques auxquels on

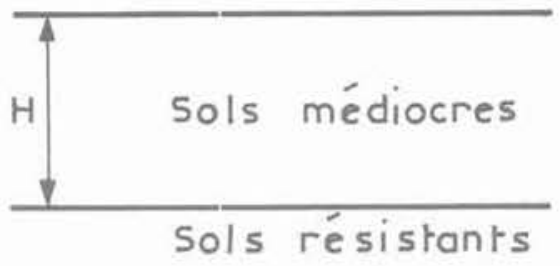

Fig. 1. - Coupe simplifiée des sols. Fig. 1. - Schematic diagram of soils.

pourra toujours ramener le réseau, de maillage géné. ralement quasi-carré, ou triangulaire (fig. 2).

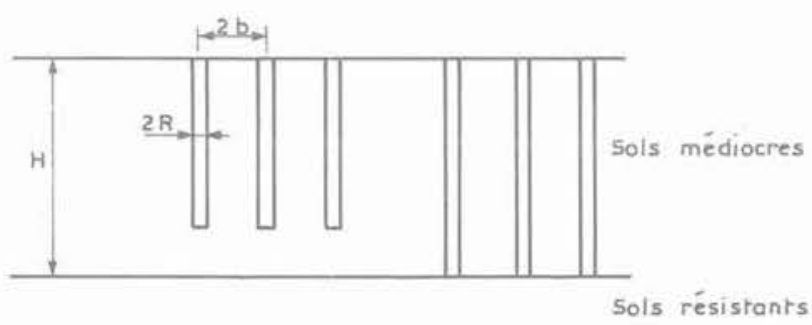

Fig. 2. - Schéma du réseau d'inclusions.

Fig. 2. - Diagram of piles network.

Le sol médiocre ainsi renforcé reçoit en surface (fig. 3) des charges de typologie variable, pouvant consister, sans que cette énumération soit exhaustive, en:

- remblai de grande dimension,

- dallage ou radier, de grande dimension, souple en général,

- semelle, éventuellement radier, rigide.

Dans les deux derniers cas, dallage ou semelle, ne sont pas solidaires des réseaux d'inclusions; ils reposent sur le sol amélioré par l'intermédiaire d'un matelas intercalaire d'épaisseur limitée, en matériau de bonne qualité, qui donne une certaine souplesse à l'ensemble. Le cas d'une semelle ou d'un radier solidaire des inclusions rigides constitue une méthode de fondation de principe et de justification différents, puisqu'il s'agit de fondations mixtes ou de radier-pieux. Une conception de dallage ou radier souple qui serait solidaire du réseau rigide relèverait, quant à elle, de l'erreur de conception, le bon sens impliquant de prévoir une couche intermédiaire " souple «, évoquée ci-dessus.

Le schéma suivant résume donc les trois situations qui viennent d'être décrites (fig. 3).

Le rôle du réseau est bien sûr d'améliorer globalement la qualité du sol de fondation; il se traduira par la possibilité, dans le cas 1 , de construire un remblai avec des tassements réduits (et donc d'économiser des matériaux), dans le cas 2 , de pouvoir édifier une structure souple (un réservoir par exemple) avec des déformées restant admissibles, et dans le cas 3 de réduire les tassements à des valeurs très faibles. 
1

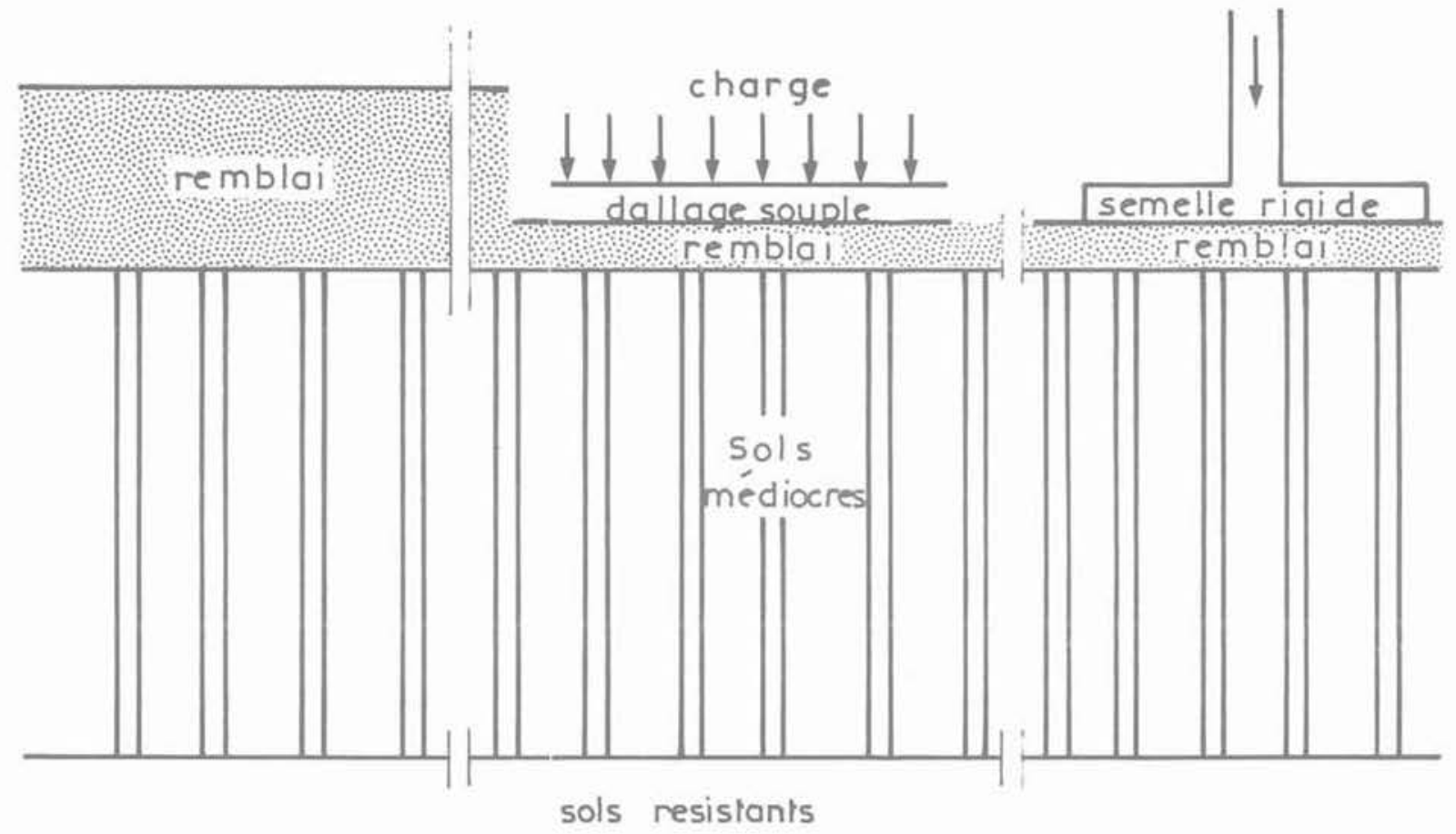

Fig. 3. - Type de constructions sur sols renforcés. Fig. 3. - Constructions on reinforced soils.

\subsection{Principe de fonctionnement}

\section{de l'inclusion isolée}

Il est qualitativement aisé de comprendre le mécanisme de fonctionnement des inclusions. La méthode de calcul proposée plus loin concernant le cas 1 (édification de remblai), nous raisonnerons à partir de ce schéma, le raisonnement restant le même, légèrement plus complexe, pour les situations 2 et 3 .

Considérons une épaisseur $\mathrm{H}$ de sol compressible, chargé par un remblai qui transmet la contraine $\mathrm{q}_{\text {; }}$; en situation finale, il s'est produit un tassement $W_{0}$ (o) à la surface, et le sol en place, en profondeur, accuse un tassement $W_{n}(z)$ illustré en 2 bis (fig. 4).

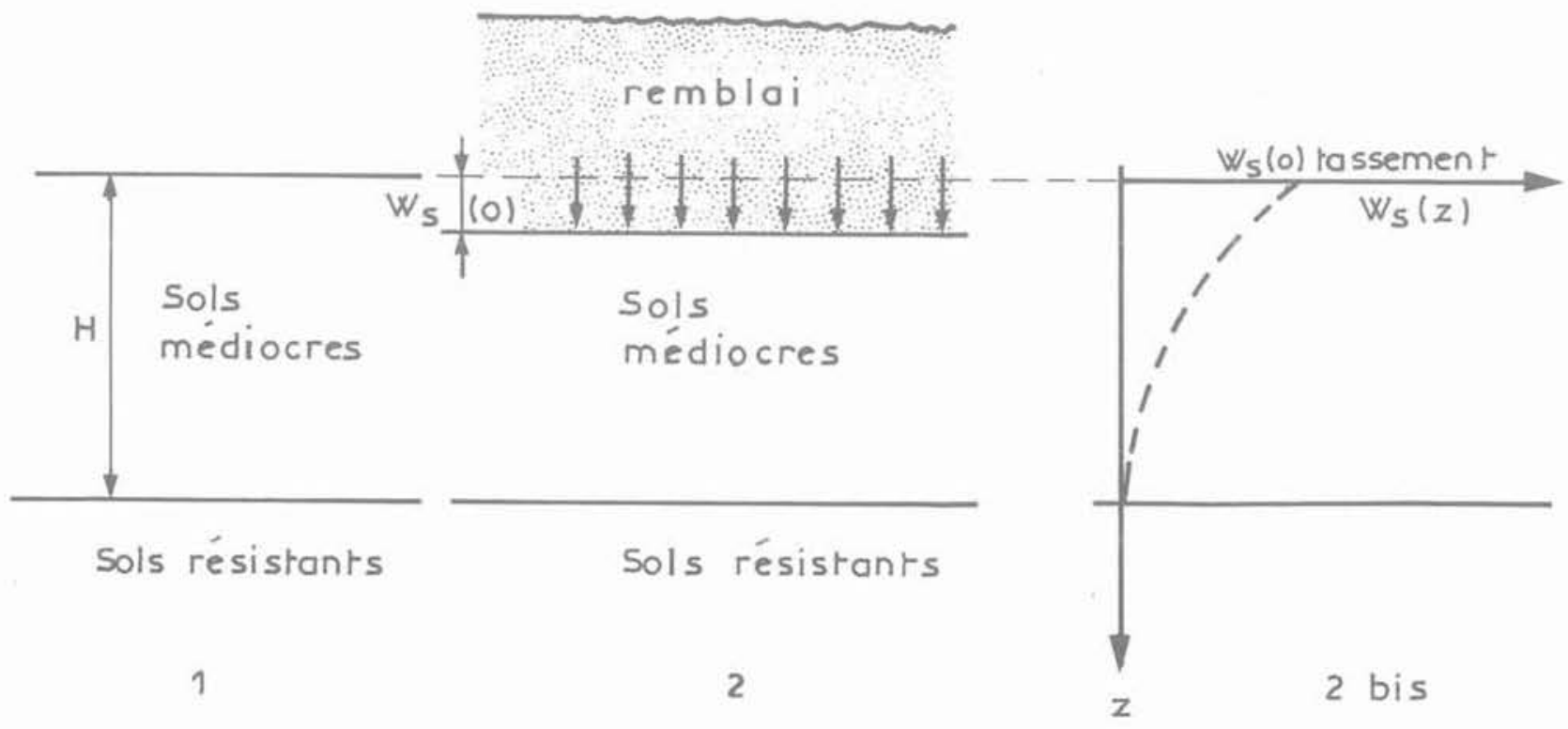

A

Fig. 4. - Sols médiocres chargés.

Fig. 4. - Loaded soft soils. 
Plaçons maintenant une inclusion rigide, seule, noyée dans le sol mou, et examinons ses conditions d'équilibre, après stabilisation complète (fin de consolidation) (fig. 5 et 6 ).

Loin de l'inclusion isolée, le comportement du sol est identique à A. Par contre, dans l'environnement im- médiat de celle-ci, il y a modification des champs de contrainte et déformation. L'inclusion subit un tassement $W_{p}(\mathrm{z})$ auquel s'ajoute une légère compression, car elle n'est pas infiniment rigide; ce tassement est évidemment plus élevé dans le cas B que dans le cas $\mathrm{C}$, où l'inclusion repose sur le sol dur. Dans la partie inférieure, le tassement du sol est inférieur à celui de

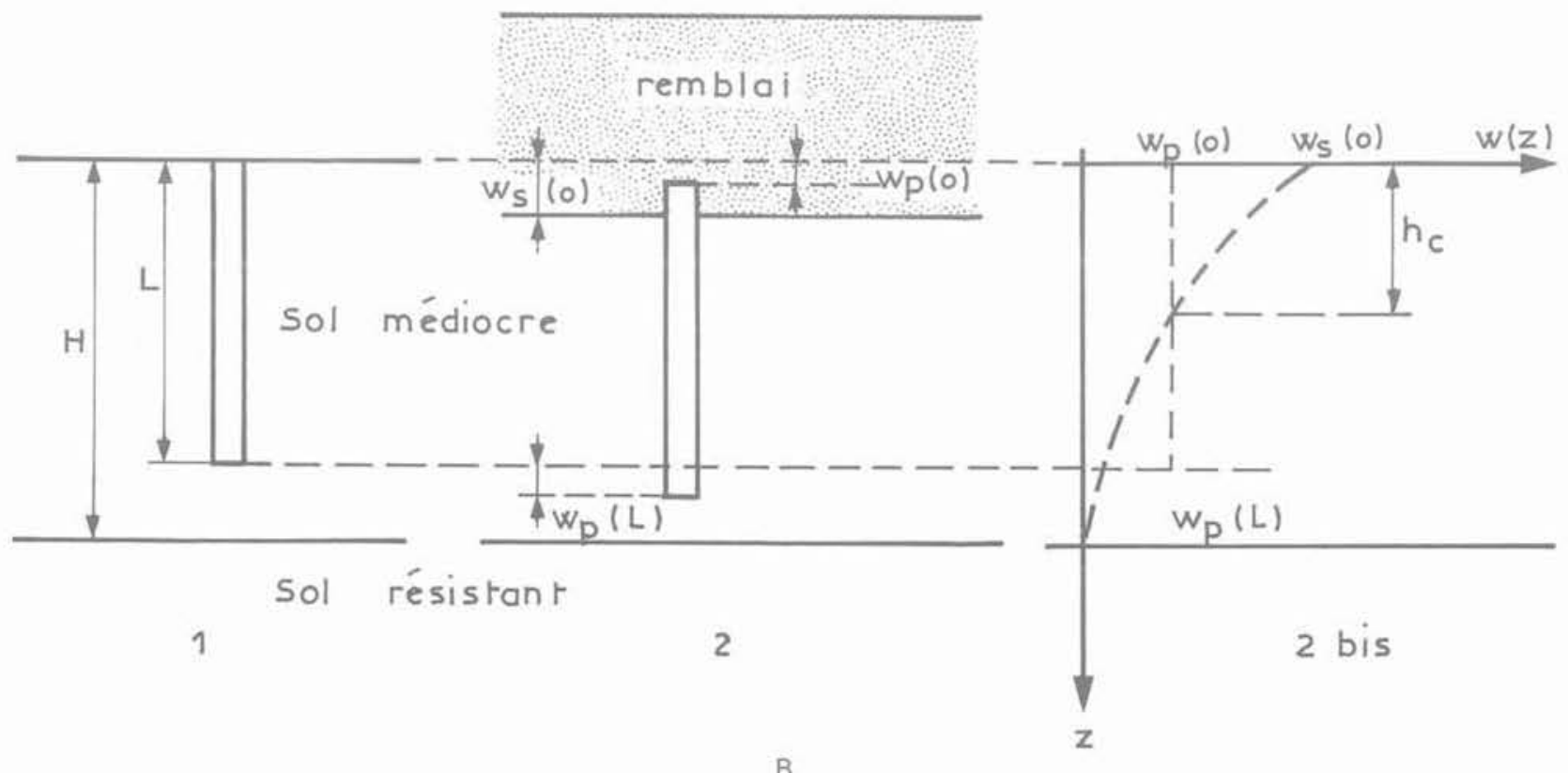

Fig. 5. - Inclusion arrêtée dans le sol médiocre.

Fig. 5. - Rigid pile in soft soils.

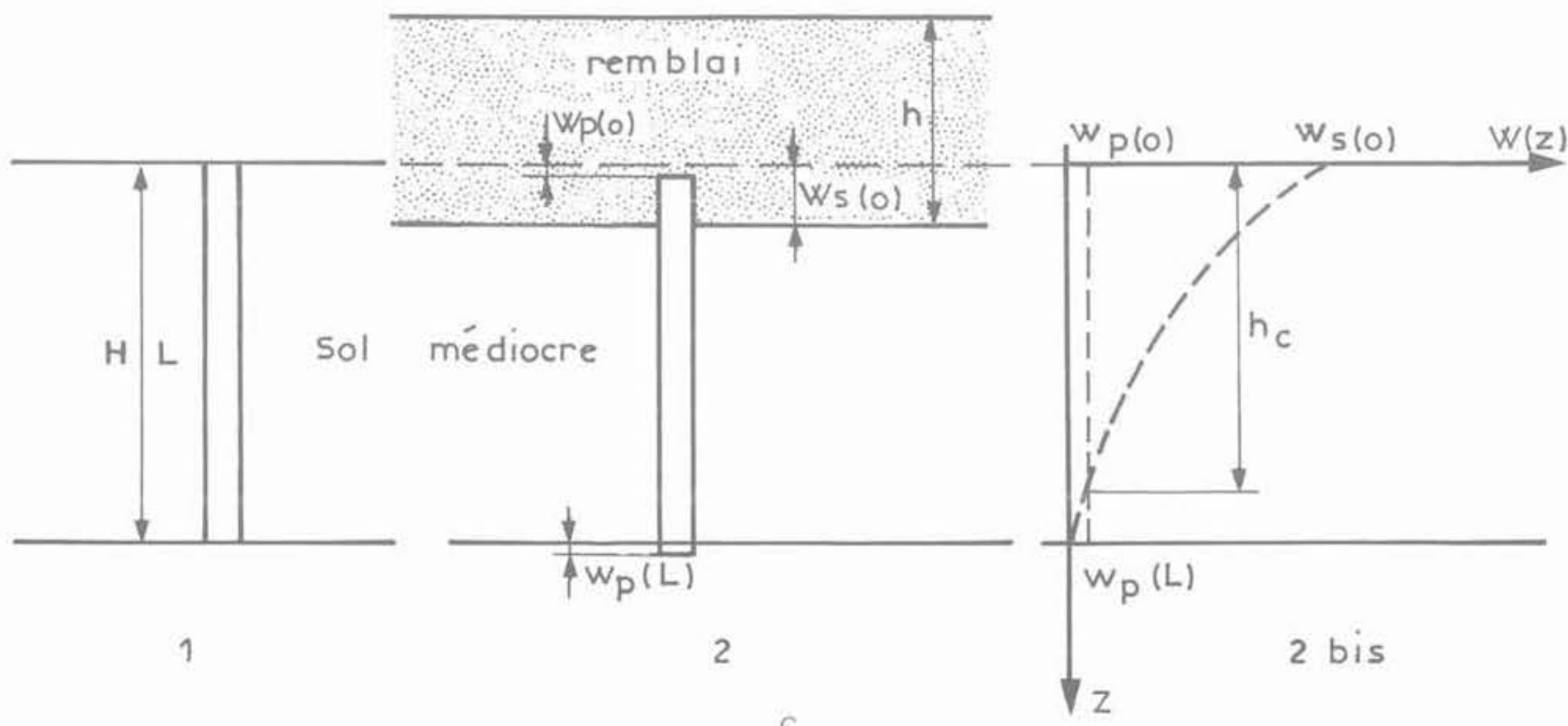

Fig. 6. - Inclusion arrêtée dans le sol résistant. Fig. 6. - Rigid pile in hard soil. 
linclusion, la configuration inverse apparaissant dans la partie supérieure (le tassement du sol, est bien entendu le tassement « libre », c'est-à-dire sans inclusion).

En tête de linclusion, celle-ci émerge du sol médiocre de la quantité $W_{0}(s)-W_{0}(p)$ et poinçonne le remblai. A ce niveau, le comportement de la face supérieure, que nous supposerons horizontale, est identique à celui d'une plaque d'ancrage, noyée à la profondeur $h$, (épaisseur du remblai), et sollicitée par traction vers lé haut. Il $y$ a donc mobilisation d'un effort, dépendant de $h_{\text {r }}$ et de la qualité du matériau constituant le remblai.

En définitive, à l'équilibre, les efforts mobilisés tout le long de l'inclusion ont quatre composantes (fig. 7):

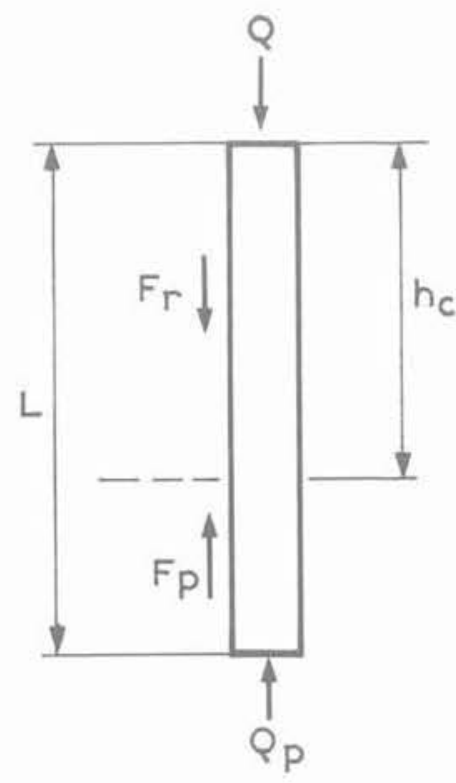

Fig. 7. - Efforts agissant sur l'inclusion.

Fig. 7. - Loads acting on pile.

efforts moteurs: ce sont la charge verticale $Q$ sur la tête, assimilable à un ancrage, et la résultante $\mathrm{F}$ des frottements de type négatif, agissant sur une longueur $\mathrm{h}$ de l'inclusion, inférieure à la longueur totale de celleci;

efforts résistants: ce sont la résultante $F_{p}$ des frottements de type positif, mobilisés en partie basse, sur la longueur $L-h$, et l'effort de pointe $Q_{\text {à la }}$ base de l'inclusion. On a bien sûr l'égalité $\mathrm{Q}+\mathrm{F}_{\mathrm{n}}=$ $F_{p}+Q_{p}$

De ce schéma de fonctionnement, on peut déduire la condition optimale d'efficacité de l'inclusion, qui est de lui transmettre les efforts moteurs maximaux, pour, de la sorte, décharger le terrain immédiatement environnant. Si l'on imagine une inclusion très lisse ne permettant pas de frottement $\left(F_{n}=F_{p}=0\right)$, ou, si l'on fait l'hypothèse approximative $F_{n}={ }^{n} F$, alors $Q=Q$; la possibilité de mobiliser $Q$, effort 'd'ancrage induit donc la nécessité de pouvoir mobiliser le même effort de signe opposé, en pointe. Cette potentialité est donc liée à la qualité du sol en pointe; on a donc tout intérêt à prévoir d'arrêter cette pointe sur un niveau suffisamment porteur. L'effort $Q$ en pointe sera d'ailleurs limité à la charge limite $\mathrm{Q}_{\mathrm{p}}$. Dans ces conditions, P'inclusion constitue un point dur, presque fixe, qui permet effectivement une mobilisation d'un effort d'ancrage optimal en tête. Bien entendu, si l'inclusion est arrêtée dans les sols médiocres, la valeur de $Q_{p l}$ mobilisable est faible, et l'inclusion se déformant alors fortement, la déformée relative sol-inclusion en tête, obligatoirement faible, ne permet qu'un faible effort d'ancrage.

D'ailleurs, à l'extrême, toujours dans lhypothèse de frottements nuls, si l'on imagine un effort de pointe nul, la tête de l'inclusion suit exactement le mouvement du sol chargé par le remblai, et aucun effort ne peut lui être transmis.

De même, imaginons une inclusion dont la longueur $\mathrm{L}$ tend vers zéro; c'est, à la limite, un disque noyé à l'interface sol-remblai, tassant comme le sol, n'encaissant aucun effort, hormis le poids des terres le surmontant, lequel est retransmis immédiatement au sol par la face inférieure du disque. $\mathrm{Si}$ l'on bloque le mouvement de disque, en intercalant entre la face inférieure et le sol dur un élément indéformable, on recrée une inclusion intéressante, remplissant son rôle, puisqu'elle canalise l'effet d'ancrage se manifestant alors en tête. Cette inclusion differe géométriquement d'une inclusion de diamètre constant (mini pieu, pilot, pieu...); c'est, en résumé, un mini pieu (ou pilot, ou pieu) capable d'encaisser les efforts que lui transmet une platine de plus grand diamètre qui la coiffe en tête. Un tel dis. positif est facilement et effectivement concevable.

En conclusion, une inclusion rigide sera d'autant plus efficace que sa capacité portante en pointe sera élevée; on recherchera donc un niveau porteur suffisant, sans lequel il est illusoire de compter sur une sérieuse efficacité.

\subsection{Principe de fonctionnement d'un réseau d'inclusions (fig. 8)}

La mise en place d'une inclusion ne modifie que localement le champ de déformation du sol compressible; celui-ci, aux alentours immédiats de l'inclusion, se déforme moins. Un grand nombre d'inclusions rap. prochées, par accumulation des modifications locales du champ des déformations libres du sol, permet par contre une réduction généralisée de la déformation du sol, réduction d'autant plus importante que le maillage du réseau est serré. Parallèlement, bien sûr, et cette réduction de tassement en résulte, les contraites dans le sol compressible sont fortement diminuées, puisque des efforts se transmettent aux inclusions.

Les mécanismes de transmission de la charge $\mathrm{q}$ apportée par le remblai sont identiques au cas de l'inclusion isolée. Les intensités en sont seulement modifiées du fait de l'effet de groupe créé par le réseau.

Pour bien saisir l'effet de groupe, on remarquera que l'efficacité maximale du réseau peut être définie comme celle qui conduirait à un tassement nul du sol compres- 


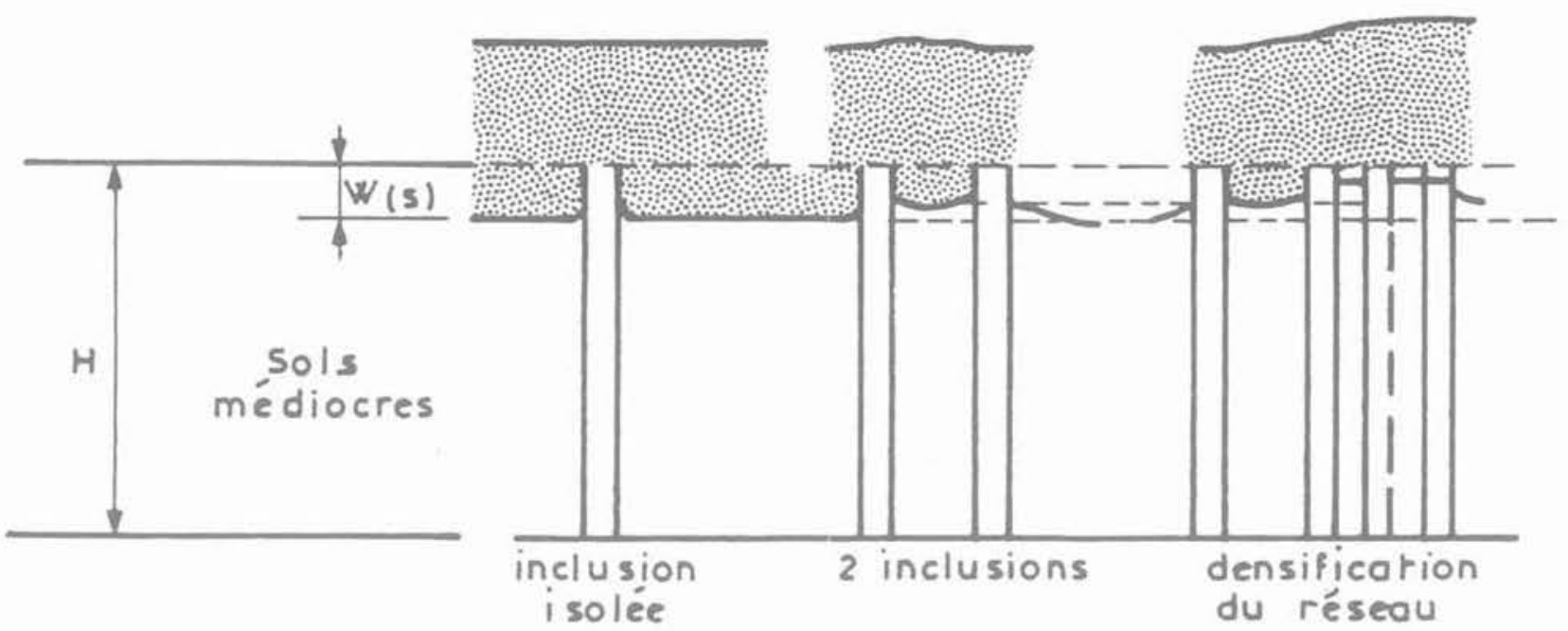

Fig. 8. - Principe de fonctionnement d'un réseau.

Fig. 8. - Working principle of a piles network.

sible après mise en place du réseau et du remblai. Ce serait un réseau d'inclusions infiniment rapprochées reprenant la totalité de la charge du remblai. Chaque inclusion (arrêtée sur le sol dur) serait finalement peu chargée, ne reprenant que le poids de la colonne de remblai la surmontant.

Nous avons vu qu'une inclusion isolée était susceptible de reprendre en tête l'effort mobilisé par l'effort d'ancrage, dont on sait qu'il est très largement supérieur au poids de la colonne de remblai le surmontant. Il apparaît donc immédiatement que l'effet de groupe présente à la fois avantage et inconvénient; avantage puisque le groupe réduit les tassements, ce qui est précisément ce que l'on cherche, et inconvénient car l'effort que peut supporter une inclusion au sein du réseau est largement inférieur à celui que l'inclusion, isolée, peut reprendre.

Un compromis sera donc à chercher pour optimiser le maillage et le dimensionnement des inclusions. On verra d'ailleurs que cette optimisation pourra conduire à prévoir une augmentation du diamètre de la tête de cellesci.

\section{MÉTHODE DE CALCUL D'UN RÉSEAU D'INCLUSIONS RIGIDES EN SOL COMPRESSIBLE}

La méthode proposée procède de la démarche suivante:

Un remblai est à édifier sur un sol compressible; il en résulte un tassement que l'on cherche à réduire par un réseau d'inclusions. Il sera pratiquement indispensable d'arrêter celles-ci dans un niveau porteur, sans lequel l'efficacité est très compromise.

Pour dimensionner le réseau, on imposera que le maillage conduise, à la surface des sols médiocres, à une contrainte « résiduelle *, que l'on se fixe, et qui est une fraction de la contrainte induite par le remblai. On calcule pour une inclusion du réseau l'effort qui lui est transmis en tête, en tenant compte de l'effet de groupe. La contrainte résiduelle à l'interface remblai-sol en place agit elle-même au niveau du fût des inclusions, qu'elle surcharge par frottement négatif. De ce fait, et par l'effet de groupe, ce frottement négatif réduit en profondeur les contraintes induites dans le sol médiocre; on tient compte de cette réduction pour le calcul du tassement final du sol compressible.

La connaissance de l'effort moteur total transmis à l'inclusion permet le dimensionnement final optimal du fût de l'inclusion; c'est de la sorte que l'on est tout naturellement amené, pour des raisons économiques, à prévoir des inclusions surmontées d'une platine beaucoup plus large que leur fût.

\subsection{Comportement en tête d'inclusion Contribution de l'effet de groupe « d'ancrage »}

Nous ne développerons pas ici les méthodes de calcul d'un ancrage isolé, auquel est assimilable la tête de l'inclusion. Ces méthodes sont variées et ont été passées en revue récemment par MORBOIS et NGUYEN THANH LONG (1984); on doit d'ailleurs y ajouter celle préconisée par MENARD (1969), conduisant à des expressions de la capacité d'ancrage identiques à celles introduites par cet auteur pour les pointes de pieux, dans la théorie pressiométrique.

Il n'existe pas de formulation en ce qui concerne l'effet de groupe. Aussi proposons nous de le modéliser comme suit, ce qui, après examen approfondi des méthodes rappelées ci-dessus et de certaines conditions aux limites concernant l'effet de groupe en question, nous a paru assez réaliste et du côté de la sécurité visà-vis du problème à résoudre: on fait l'hypothèse que l'effet de groupe des têtes d'inclusions est équivalent à 
l'effet de groupe correspondant à des éléments fictifs, ayant la même section droite que les inclusions et surchargés, sur la hauteur $\mathrm{h}$, par frottement négatif (fig. 9).

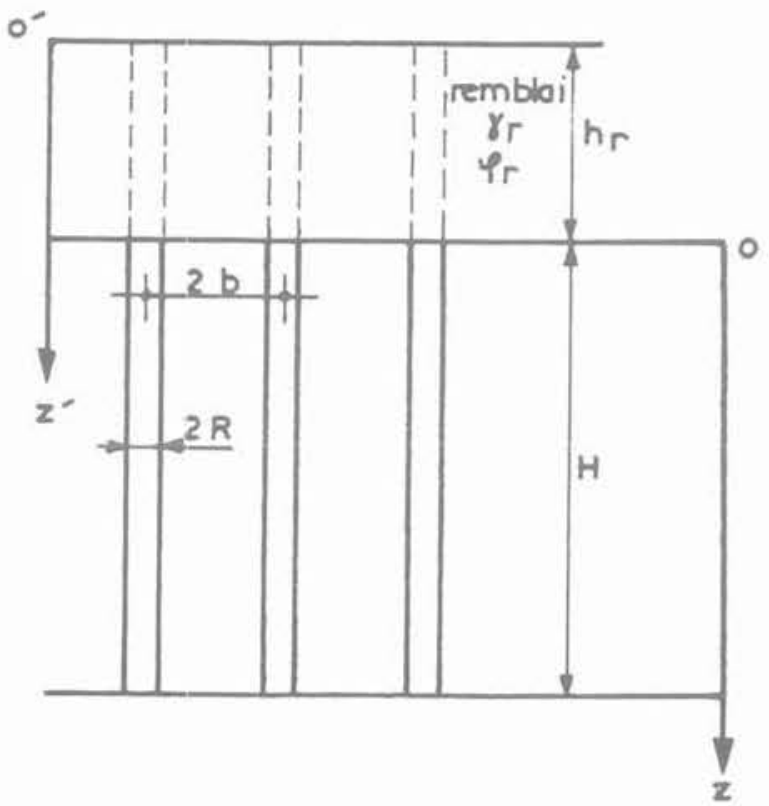

Fig. 9. - Géométrie sol renforcé-remblai.

Fig. 9. - Geometry of reinforced soil and fill.

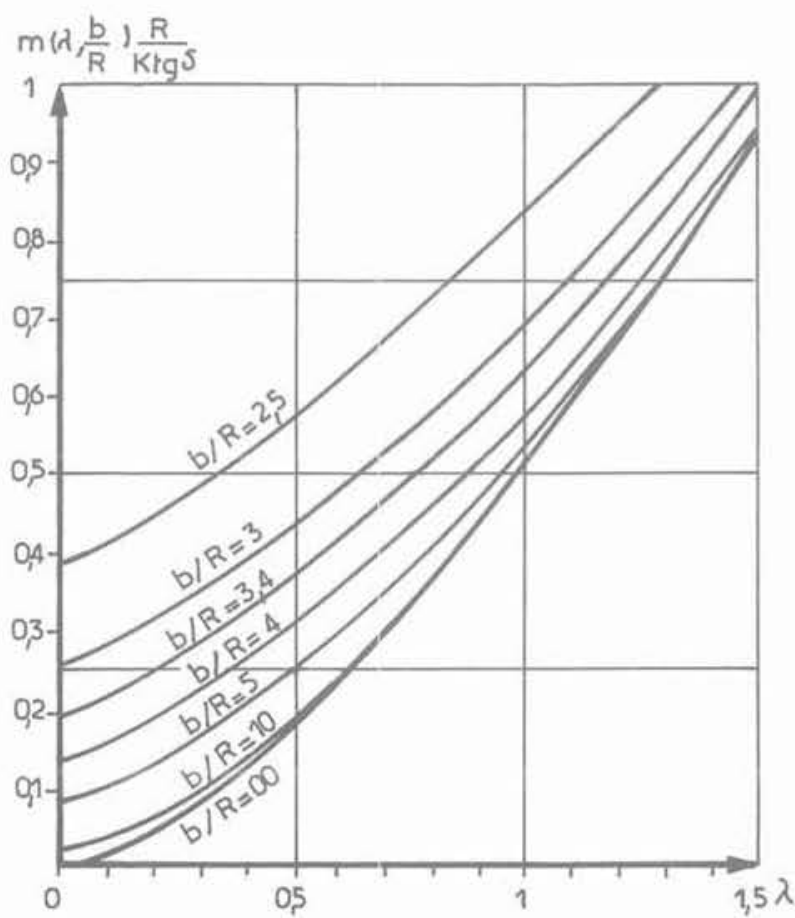

Abaque 1. - Détermination de $m\left(\lambda, \frac{b}{R}\right)$
On se reportera aux études de COMBARIEU (1974, 1985) sur le frottement négatif, qui définissent tous les paramètres utilisés pour la justification de la méthode exposée et à laquelle il est fait très largement appel.

Dans les conditions posées, la contrainte verticale $\mathrm{q}^{\prime}\left(z^{\prime}\right)$ qui règne à la cote $z^{\prime}$ dans le remblai, entre les inclusions fictives, est donnée par

$$
q^{\prime}\left(z^{\prime}\right)=\frac{\gamma_{r}}{m_{r}}\left(1-e^{-m_{r z}}\right)
$$

où $\lambda$ et $\mathrm{m}\left(\lambda, \frac{\mathrm{b}}{\mathrm{R}}\right)$ sont successivement déterminés comme suit :

Le remblai a un angle de frottement $\varphi$; le long des inclusions fictives, le contact est caracterisé par Ktg $\delta$ (avec $\delta=\varphi_{t}$ ), que l'on se fixe.

Le coefficient d'accrochage $\lambda$ est donné par l'une des expressions suivantes:

$\begin{array}{ll}\lambda=\frac{1}{0,5+25 \mathrm{~K} \operatorname{tg} \delta} \text { pour } \operatorname{Ktg} \delta \leqslant 0,15 \\ \lambda=0,385-\mathrm{K} \operatorname{tg} \delta & \text { pour } 0,15 \leqslant \mathrm{~K} \operatorname{tg} \delta \leqslant 0,385 \\ \lambda=0 & \text { pour Ktg } \delta \geqslant 0,385\end{array}$ $m\left(\lambda, \frac{b}{R}\right) \frac{R}{k \lg S}$

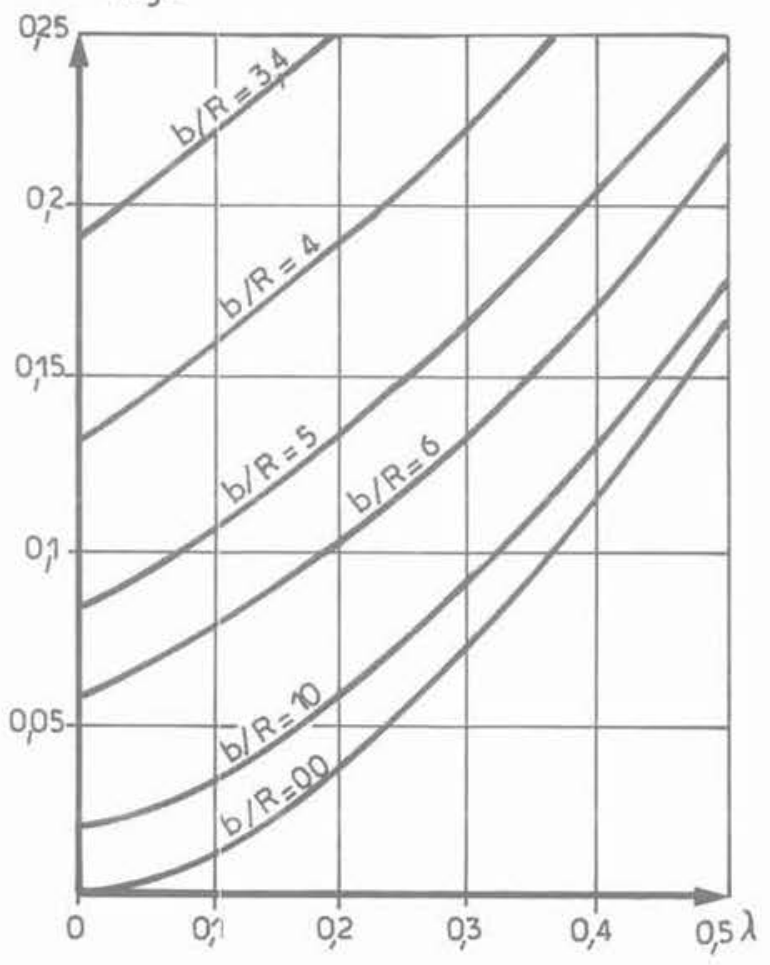

Graph 1. - Determination of $m\left(\lambda, \frac{b}{R}\right)$ 
Le coefficient $m\left(\lambda, \frac{b}{R}\right)$, est déterminé en utilisant l'abaque 1 , connaissant $\frac{b}{R}, R, K \operatorname{tg} \delta$. Pour le remblai, où l'on aura généralement $\lambda=0$, on obtient:

$$
\mathrm{m}\left(\lambda=0, \frac{\mathrm{b}}{\mathrm{R}}\right)=\frac{2 \mathrm{R} \operatorname{Ktg} \delta}{\mathrm{b}^{2}-\mathrm{R}^{2}}
$$

Si le maillage est carré, avec une maille $\mathrm{d}$, on a

$\frac{\mathrm{b}}{\mathrm{R}}=\frac{\mathrm{d}}{\mathrm{R}} \sqrt{\frac{4}{\pi}}$

$\frac{b}{R}=\frac{1}{R} \sqrt{\frac{4}{\pi} \mathrm{dd}^{\prime}}$

s'il est rectangulaire, alors

Au niveau $z^{\prime}=h$ de la base du remblai, (où $z=0$ pour le sol naturel), la contrainte résiduelle vaut donc:

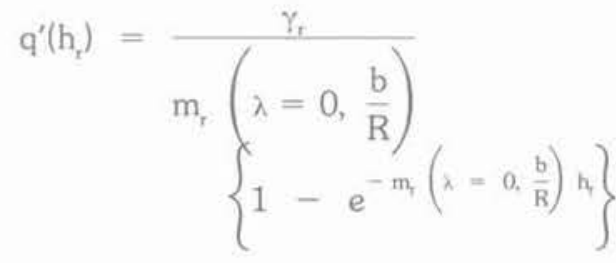

Finalement, au niveau $\mathrm{z}=\mathrm{o}$, on a l'égalité :

$$
\begin{aligned}
\pi b^{2} \gamma_{r} h_{r}=\pi\left(b^{2}-R^{2}\right) & q^{\prime}\left(h_{r}\right) \\
& +\pi R^{2} \gamma_{r} h_{r}+F_{n_{s}}
\end{aligned}
$$

où $\mathrm{F}_{\mathrm{nt}}$ est le frottement négatif transmis à l'inclusion, laquelle est chargée par $\mathrm{Q}=\mathrm{F}_{\mathrm{n}, \mathrm{e}}+\pi \mathrm{R}^{2} \gamma_{r} \mathrm{~h}_{r}$ ( 2 bis), valeur que l'on cherchera à rendre la plus forte possible, de sorte que la contrainte $\mathrm{q}^{\prime}(\mathrm{h})$ soit, elle, la plus petite possible.

En pratique, on se fixera comme objectif de ramener sur le sol médiocre la contrainte $\gamma, \mathrm{h}$, sans renforcement à une valeur q' $\left(h_{r}\right)$, après renforcement, acceptable visà-vis des tassements ultérieurs qu'elle engendrera.

On pose donc $\mathrm{q}^{\prime}\left(\mathrm{h}_{\mathrm{f}}\right)=\alpha \gamma \mathrm{h}$, (3), définissant le paramètre $\alpha<1$, et la liberté de choix est laissée quant à la valeur du diamètre $2 \mathrm{R}$ de l'inclusion; on en déduira $\frac{b^{2}}{R^{2}}$, donc le maillage. D'après (1) et (3), on a $\alpha \gamma_{r} h_{r}=\frac{\gamma_{r}}{m_{r}}\left(1-e^{-m_{r} h_{r}}\right)$ soit $e^{-m_{1} h_{r}}=1-\alpha m_{r} h_{r}$ (4) qui déterminera $m_{r}$ $h_{r}(\alpha)$ pour $\alpha$ donné (abaque 2 ).

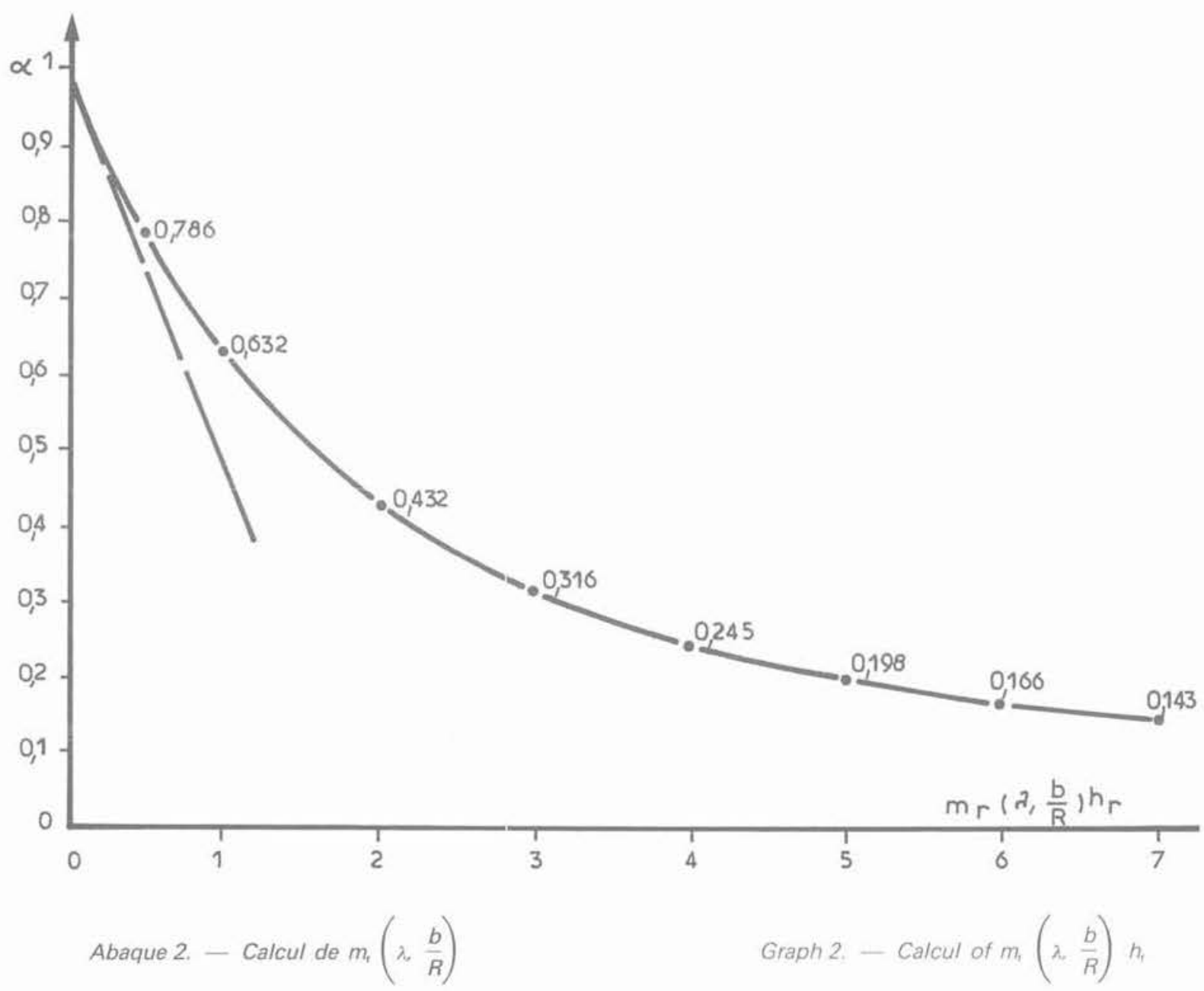


Le maillage est donné par (abaque 3):

$$
\frac{b^{2}}{\mathrm{R}^{2}}=1+\frac{2 \mathrm{Ktg} \varphi_{r}}{\left[\mathrm{~m}_{t}\left(\lambda, \frac{b}{\mathrm{R}}\right) \cdot \mathrm{h}_{t}\right]_{(\mathrm{s})}} \cdot \frac{\mathrm{h}_{r}}{\mathrm{R}}
$$

Ce résultat mène aux constatations suivantes, conformes aux conclusions auxquelles on peut aboutir par un raisonnement qualitatif :

1. à qualité croissante de remblai, l'intensité du maillage nécessaire décroît. L'effet de voûte entre têtes d'inclusion est effectivement meilleur :

2. plus $\alpha$ est faible, plus le maillage est serré;

3. pour une valeur $\mathrm{R}$ du rayon de l'inclusion, le maillage augmente (à $a$ donné) avec $\mathrm{h}, \mathrm{q}^{\prime}\left(\mathrm{h}_{\mathrm{r}}\right.$ ) augmentant plus vite que $\gamma, h_{r}$

Une manière différente de poser le problème consiste à raisonner à partir de la charge transmise. C'est ainsi que les Scandinaves, adeptes de ce procédé, imposent qu'une fraction $\beta$ donnée de la charge totale du remblai soit transmise à la surface du sol entre les inclusions, soit $\beta \pi b^{2} \gamma_{t} h_{t}=\pi\left(b^{2}-R^{2}\right) q^{\prime}\left(h_{r}\right)$;

on peut ainsi déterminer le maillage $\frac{b^{2}}{R^{2}}$ pour $\beta$ fixé, en fonction de $\frac{h_{r}}{R}$; dans ce cas,

$$
m_{r} h_{r}\left(\beta, \frac{h_{r}}{R}\right)
$$

est donné par

$$
\begin{gathered}
e^{-\mathrm{m}_{r}\left(\lambda, \frac{\mathrm{b}}{\mathrm{R}}\right) h_{r}}=1-\beta \mathrm{m}_{r}\left(\lambda, \frac{\mathrm{b}}{\mathrm{R}}\right) \\
\mathrm{h}_{r}\left(1+\frac{\mathrm{m}_{r}\left(\lambda, \frac{\mathrm{b}}{\mathrm{R}}\right) \mathrm{h}_{r}}{2 \operatorname{Ktg}_{\varphi_{r}} \frac{\mathrm{h}_{r}}{\mathrm{R}}}\right)
\end{gathered}
$$

et

$$
\frac{\mathrm{b}^{2}}{\mathrm{R}^{2}}=1+\frac{2 \mathrm{Ktg} \varphi_{r}}{\mathrm{~m} \lambda_{1} \frac{\mathrm{b}}{\mathrm{h}} \mathrm{h}} \cdot \frac{\mathrm{h}_{\mathrm{r}}}{\mathrm{R}}
$$

et on montre sans difficulté que, lorsque $\frac{h_{1}}{R}$ tend vers $O, \frac{b^{2}}{R^{2}}$ tend vers $\frac{1}{1-\beta}$ ou, au contraire, si $\frac{h_{r}}{R}$ tend vers l'infini, la relation (6) se réduit à

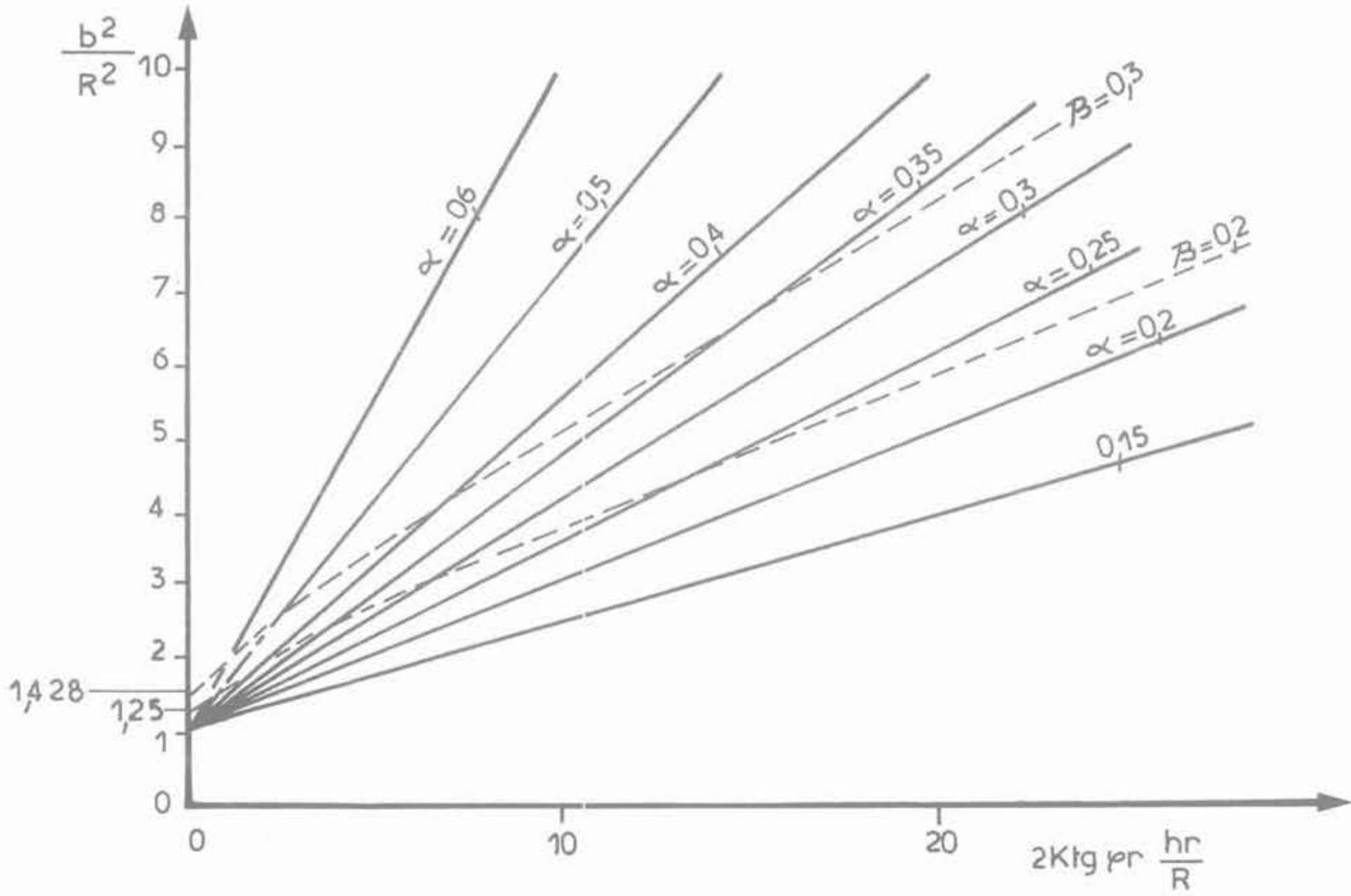

Abaque 3. - Maillage en fonction d'un critère de contrainte $(\alpha)$ ou de charge $(\beta)$

Graph 3. - Spacing of piles with stress $(\alpha)$ or load $(\beta)$ criterion. 


$$
e^{-m_{r}\left(\lambda_{1} \frac{b}{R}\right) h_{t}}=1-\beta m_{r} h_{r}
$$

analogue à (4). L'abaque 3 donne, suivant le critère choisi ( $\alpha$ ou $\beta$ ) le maillage en fonction de $2 \operatorname{Ktg} \varphi_{x} \cdot \frac{h}{R}$

\subsection{Comportement dans le sol médiocre} sous $\mathrm{q}^{\prime}\left(\mathrm{h}_{\mathrm{r}}\right)$.

\section{Contribution des effets de groupe et d'accrochage}

L'effet de voûte entre les têtes d'inclusions amène en définitive au niveau $z=0$ du sol en place la contrainte à la valeur résiduelle q' (h) (fig. 10), chaque inclusion étant soumise en tête à l'éffort $\mathrm{F}_{\pi t}+\pi \mathrm{R}^{2} \gamma, \mathrm{h}$, défini par (2) et ( 2 bis). des tassements, q $(z, x)$ est, dans l'expression ci-dessus, remplacé par une valeur moyenne uniforme $\bar{q}(\mathrm{z})$ à la cote $\mathrm{z}$, telle que

$$
2 \pi \int_{R}^{b} q(z, x) \times \lambda x=\pi\left(b^{2}-R^{2}\right) \bar{q}(z)
$$

qui conduit finalement à

$$
\bar{q}(z)=q^{\prime}\left(h_{r}\right)+\gamma^{\prime} z-
$$

$$
\left\{q^{\prime}(h)+\gamma_{r}^{\prime} z-q(z \cdot R)\right\}^{m\left(0, \frac{b}{R}\right)} \frac{m\left(\lambda, \frac{b}{R}\right)}{m(n)}
$$

où $\lambda$ est le coefficient d'accrochage du sol autour du fût, donné par le tableau 1 en fonction de Ktgo (lié au

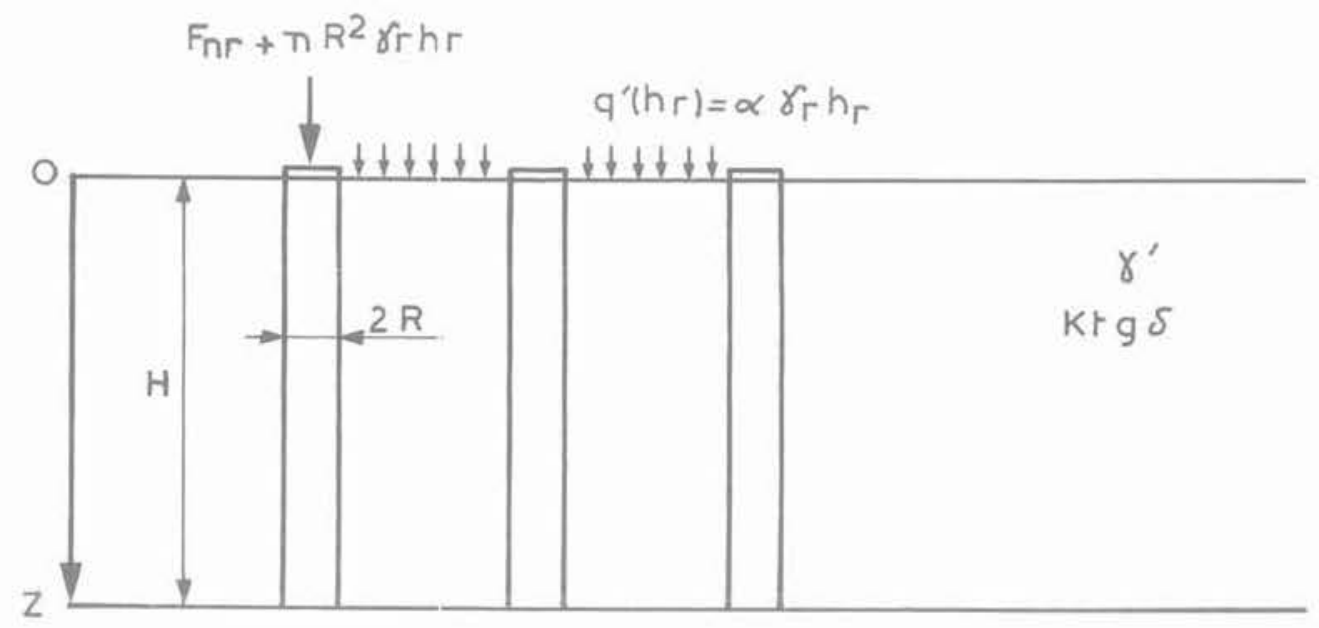

Fig. 10. - Effort en tête de pieu et contrainte résiduelle sur le sol.

Fig. 10. - Load at pile top and residual stress on soil.

Le rôle bénéfique des inclusions ne s'arrête pas à ce simple transfert en tête. Le sol médiocre, soumis à $q^{\prime}$ (h), va en effet surcharger les fûts d'inclusions par frottement négatif, lequel par effet de groupe va décharger le sol en profondeur.

Au niveau $\mathrm{z}$, on a l'égalité :

$$
\begin{aligned}
& F_{n, s}+2 \pi \int_{R}^{b} q(z, x) x \lambda d x= \\
& \pi\left(b^{2}-R^{2}\right)\left\{q^{\prime}\left(h_{r}\right)+\gamma^{\prime} z\right\}
\end{aligned}
$$

où $F_{\text {ns }}(z)$ est le frottement négatif induit par $q^{\prime}(h)$, et $\mathrm{q}(\mathrm{z}, \mathrm{x})$ la contrainte effective verticale, variable horizontalement et verticalement. Afin de permettre le calcul type de pieu et à la nature du sol); $m \quad \lambda, \frac{b}{R}$ et $\mathrm{m}\left(\mathrm{o}, \frac{\mathrm{b}}{\mathrm{R}}\right)$ sont foumis par l'abaque $1 ; \mathrm{q}(\mathrm{z}, \mathrm{R})$ est la contrainte verticale le long de l'inclusion, dans le sol médiocre, à la cote $\mathrm{z}$, valant:

$$
\begin{aligned}
& \mathrm{q}(\mathrm{z}, \mathrm{R})=\frac{\gamma^{\prime}}{\mathrm{m}\left(\lambda, \frac{\mathrm{b}}{\mathrm{R}}\right)} \\
&+\mathrm{e}^{-\mathrm{m}\left(\lambda, \frac{\mathrm{b}}{\mathrm{R}}\right)}\left\{\mathrm{q}^{\prime}\left(h_{r}\right)-\frac{\gamma^{\prime}}{\mathrm{m}\left(\lambda, \frac{\mathrm{b}}{\mathrm{R}}\right)}\right\}
\end{aligned}
$$


La connaissance de $\bar{q}(\mathrm{z})$ permet le calcul du tassement à tout niveau.

On distingue deux cas, nécessitant le calcul préalable de la hauteur maximale $h$ d'action du frottement négatif $F_{n s}$, définie en écrivant que la contrainte $q\left(h_{c}, R\right)$ calculée par (9) est égale au « poids déjaugé des terres * $\gamma^{\prime} h_{c}$.

L'abaque 4 permet directement cette détermination, à $\mathrm{m}\left(\lambda, \frac{\mathrm{b}}{\mathrm{R}}\right) \cdot \mathrm{q}^{\prime}\left(\mathrm{h}_{\mathrm{r}}\right)$

partir des termes

$$
\mathrm{m}\left(\lambda, \frac{\mathrm{b}}{\mathrm{R}}\right) \cdot \mathrm{q}^{\prime}\left(\mathrm{h}_{\mathrm{r}}\right)
$$

$\mathrm{m}\left(\lambda, \frac{\mathrm{b}}{\mathrm{R}}\right) \cdot \mathrm{h}_{\mathrm{c}}$, puis $\mathrm{h}_{\mathrm{c}}$. donnant

$I^{e r}$ cas : $h_{c} \leqslant \mathrm{H}$; le frottement négatif $\mathrm{F}_{n_{s}}$ se manifeste jusqu'à $h$, profondeur du « point neutre ». Jusqu'à cette cote, $\bar{q}(z)$ est donné par (8). A la cote $h$, on a

$$
\bar{q}\left(h_{c}\right)=\gamma^{\prime} h_{c}+q^{\prime}\left(h_{c}\right)\left\{1-\frac{m\left(o, \frac{b}{R}\right)}{m\left(\lambda, \frac{b}{R}\right)}\right\}
$$

- Si, par exemple, par simplification pour le calcul des tassements et dans la mesure où cette simplification est justifiée on choisit une contrainte moyenne sur la hauteur $h_{c}$,

soit

$$
\overline{\overline{\mathrm{q}}}=\frac{1}{\mathrm{~h}_{\mathrm{c}}} \int_{0}^{\mathrm{h}_{\mathrm{f}}} \overline{\mathrm{q}}(\mathrm{z}) \mathrm{dz}
$$

on montre que

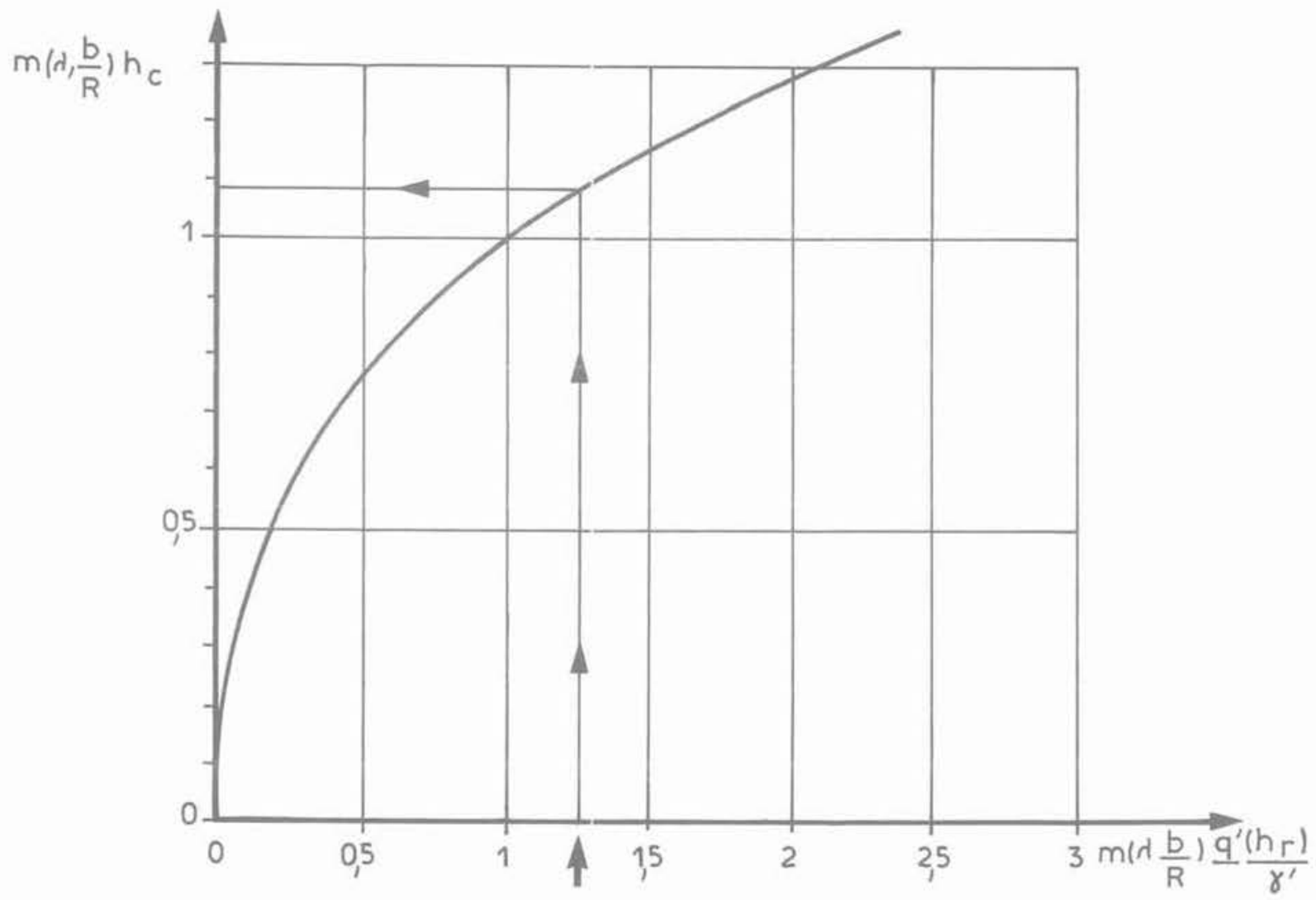

Abaque 4. - Détermination de la hauteur critique $h_{\mathrm{c}}$ dans un sol homogène chargé uniformément.

Graph 4. - Calcul of critical length he in uniformly loaded homogeneous soil. 


$$
\begin{gathered}
\overline{\overline{\mathrm{q}}}=\left\{1-\frac{\mathrm{m}\left(0, \frac{\mathrm{b}}{\mathrm{R}}\right)}{\mathrm{m}\left(\lambda, \frac{\mathrm{b}}{\mathrm{R}}\right)}\right\} \\
\left\{\gamma^{\prime} \frac{\mathrm{h}_{c}}{2}+\mathrm{q}^{\prime}\left(\mathrm{h}_{\mathrm{r}}\right)\right\}+\frac{\mathrm{m}\left(0, \frac{\mathrm{b}}{\mathrm{R}}\right)}{\mathrm{m}^{2}\left(\lambda, \frac{\mathrm{b}}{\mathrm{R}}\right)} \cdot \frac{\mathrm{q}^{\prime}\left(\mathrm{h}_{\mathrm{r}}\right)}{\mathrm{h}_{c}}
\end{gathered}
$$

Le tassement est alors donné (sol homogène normalement consolidé) par

$$
\frac{\Delta h_{c}}{h_{c}}=\frac{C_{c}}{1+e_{o}} \log \frac{2 \overline{\bar{q}}}{\gamma^{\prime} h_{c}}
$$

L'épaisseur inférieure $\mathrm{H}-\mathrm{h}$ de sol médiocre est sollicité par $\overline{\mathrm{q}}\left(\mathrm{h}_{\mathrm{c}}\right)$ donné en $(10)$; son tassement est, toujours en supposant la simplification justifiée:

$$
\begin{gathered}
\frac{\Delta\left(H-h_{c}\right)}{H-h_{c}}=\frac{C_{c}}{1+e_{o}} \log \\
\left\{1+\frac{2 q^{\prime}\left(h_{r}\right)}{\gamma^{\prime}\left(H+h_{c}\right)} \cdot \frac{1-m\left(0, \frac{b}{R}\right)}{1-m\left(\lambda, \frac{b}{R}\right)}\right.
\end{gathered}
$$

$2^{\circ}$ cas: $h_{c}>\mathrm{H}$; il n'y a donc pas de point neutre; dans ce cas, avec la même simplification qu'en (11), on a :

$$
\begin{aligned}
\overline{\bar{q}}= & \left\{1-\frac{\mathrm{m}\left(0, \frac{\mathrm{b}}{\mathrm{R}}\right)}{\mathrm{m}\left(\lambda, \frac{\mathrm{b}}{\mathrm{R}}\right)}\right\}\left\{\gamma^{\prime} \frac{\mathrm{H}}{2}+\mathrm{q}^{\prime}\left(\mathrm{h}_{r}\right)\right\} \\
+ & \frac{\mathrm{m}\left(\mathrm{o}, \frac{\mathrm{b}}{\mathrm{R}}\right)}{\mathrm{m}^{2}\left(\lambda, \frac{\mathrm{b}}{\mathrm{R}}\right)}\left\{\gamma^{\prime}+\frac{\mathrm{q}^{\prime}\left(\mathrm{h}_{r}\right)}{\mathrm{H}}-\frac{\mathrm{q}(\mathrm{H}, \mathrm{R})}{\mathrm{H}}\right\}
\end{aligned}
$$

et le tassement de la couche $\mathrm{H}$ vaut

$$
\frac{\Delta H}{H}=\frac{C_{c}}{1+e_{0}} \log \frac{2 \overline{\bar{q}}}{\gamma^{\prime} H}
$$

Il y a évidemment, dans le cas de sols variés, possibilité de tenir compte de la stratigraphie dans les calculs des contraintes et tassements. En sol homogène, on peut découper le terrain en couches médiocres au sein desquelles on pourra procéder à des calculs de tassement plus précis que la méthode approximative présentée. Ce sera absolument nécessaire dans le cas de forte épaisseur $H$, pour éviter des erreurs importantes sur l'amplitude des déformations (voir annexe).

Remarque: A l'hypothèse d'un accrochage maximum le long des pieux $(\lambda=0)$, donc d'un frottement négatif lui-même maximum, correspond la contrainte $\overline{\mathrm{q}}(\mathrm{z}) \mathrm{mi}$ nimale calculée, et en conséquence les tassements minimaux. L'accrochage $\lambda>0$ manifeste donc des effets dans le sens de la sécurité vis-à-vis du problème que l'on cherche à traiter, il est donc nécessaire de le prendre en compte. Quantitativement, son effet apparait

$$
\text { par le terme } \frac{m\left(\left(0, \frac{b}{R}\right)\right.}{m\left(\lambda, \frac{b}{R}\right)} \text {, dans les expressions (8) et }
$$

suivantes.

\subsection{Effort total $Q_{1}$ dans une inclusion}

Le frottement négatif induit le long de l'inclusion dans le sol mou est donné par

$$
F_{n, s}=\frac{2 \pi R K \operatorname{tg} \delta}{m\left(\lambda, \frac{b}{R}\right)}\left\{\gamma^{\prime} H+q^{\prime}\left(h_{r}\right)-q(H, R)\right\}
$$

s'il n'y a pas de point neutre, et par

$$
\mathrm{F}_{\mathrm{ns}}=\frac{2 \pi \mathrm{RK \operatorname {tg } \delta}}{\mathrm{m}\left(\lambda, \frac{\mathrm{b}}{\mathrm{R}}\right)} \mathrm{q}^{\prime}\left(\mathrm{h}_{\mathrm{r}}\right)
$$

s'il $y$ a un point neutre.

Donc, globalement, l'inclusion doit être dimensionnée pour reprendre l'effort total

$$
Q_{t}=\pi R^{2} \gamma_{r} h_{r}+F_{n, t}+F_{n s}
$$

s'écrivant

$$
\begin{aligned}
Q_{t}= & \pi b^{2} \gamma_{r} h_{r}-\pi\left(b^{2}-R^{2}\right) q^{\prime}\left(h_{r}\right) \\
+ & \frac{2 \pi R K_{t g} \delta}{\left(\lambda, \frac{b}{R}\right)}\left\{\gamma^{\prime} H+q^{\prime}\left(h_{r}\right)-q(H, R)\right\}
\end{aligned}
$$


ou bien

$$
\begin{aligned}
Q_{1}=\pi b^{2} \gamma_{r} h_{1}-\pi\left(b^{2}-\right. & \left.R^{2}\right) q^{\prime}\left(h_{r}\right) \\
+ & \frac{2 \pi R \operatorname{Rtg} \delta}{\left(\lambda, \frac{b}{R}\right)} \cdot q^{\prime}\left(h_{r}\right)
\end{aligned}
$$

où $\mathrm{q}(\mathrm{H}, \mathrm{R})$ est défini en (9).

Comme on l'a postulé en début de cette étude, les termes $F_{\text {ini }}$ et $F_{\text {is }}$ ne se développent que si le déplacement relatif sol-inclusion est suffisant, ce qui nécessite donc un sol suffisamment compressible. Pour le type d'applications examiné ici, ce sera toujours le cas; il y a également nécessité, d'un sol assez peu déformable sous la pointe de pieu.

On dimensionnera donc les inclusions pour la valeur $Q_{4}$ calculée ci-dessus, ce qui implique en pratique la présence d'un horizon de portance suffisante à distance modérée.

On pourra bien entendu choisir, vis-à-vis de la charge limite $\mathrm{Q}_{\mathrm{pl}}$ en pointe de l'inclusion, un coefficient de sécurité relativement faible, menant à une charge ne restant que légèrement inférieure à la charge de fluage $Q_{F}$.

En effet, imaginons que l'on dimensionne l'inclusion de sorte que la charge $\mathrm{Q}$ approche $\mathrm{Q}_{\mathrm{pl}}$. La pointe va de ce fait tasser et les conditions d'une mobilisation optimale du frottement négatif le long du fût n'étant pas respectées (point fixe), le terme $F_{n s}$ réel sera plus faible que celui calculé, tout en ayant en tête un déplacement relatif suffisant pour mobiliser $\mathrm{F}_{n \mathrm{r}}$. En définitive, dans de telles conditions, chaque inclusion n'encaisserait en réalité, après redistribution, qu'un effort inférieur à $Q$ prévu; la différence bien entendu serait retransmise au sol médiocre. Il y a d'ailleurs possibilité de chiffrer ce transfert par application des méthodes générales déjà citées (COMBARIEU 1985), qui prennent en compte une hauteur réduite d'action $h_{c}^{\prime}<h_{c}$ de frottement négatif.

Dans le cas où les inclusions seraient arrêtées au sein même du sol médiocre, on pourra procéder comme suit pour chiffrer leur capacité d'amélioration.

1. On fait toujours l'hypothèse qu'il y a un déplacement relatif sol-pieu suffisant en tête d'inclusion pour mobiliser $F_{\text {t }}$ par effet d'ancrage. On en déduit le maillage. On estime, pour les inclusions, la hauteur d'action réduite h'c, et l'on procède au calcul des tassements des épaisseurs $h_{c}^{\prime}$ et $\mathrm{H}-h^{\prime}$, comme précédemment. Chaque inclusion est alors soumise, à la profondeur $h^{\prime}$ à un effort qui a pour intensité $Q_{1}=\pi b^{2} \gamma_{t}+$ $\mathrm{F}_{n+}+\mathrm{F}_{n \text { s. }}$. Cet effort, le pieu étant arrêté dans le sol médiocre, est retransmis intégralement à celui-ci par frottement latéral positif et en pointe de l'inclusion.

Il contribue donc de ce fait à un tassement supplémentaire de la couche $\mathrm{H}-h^{\prime}$, que l'on pourra apprécier par la méthode de TERZAGHI, par exemple, en sup. posant que l'effort $Q_{1}$ est reporté sur une semelle fictive située à la profondeur $h_{c}^{\prime}+\frac{2}{3}\left(L-h_{c}^{\prime}\right),(L$, longueur de pieu < H) (fig. 11).

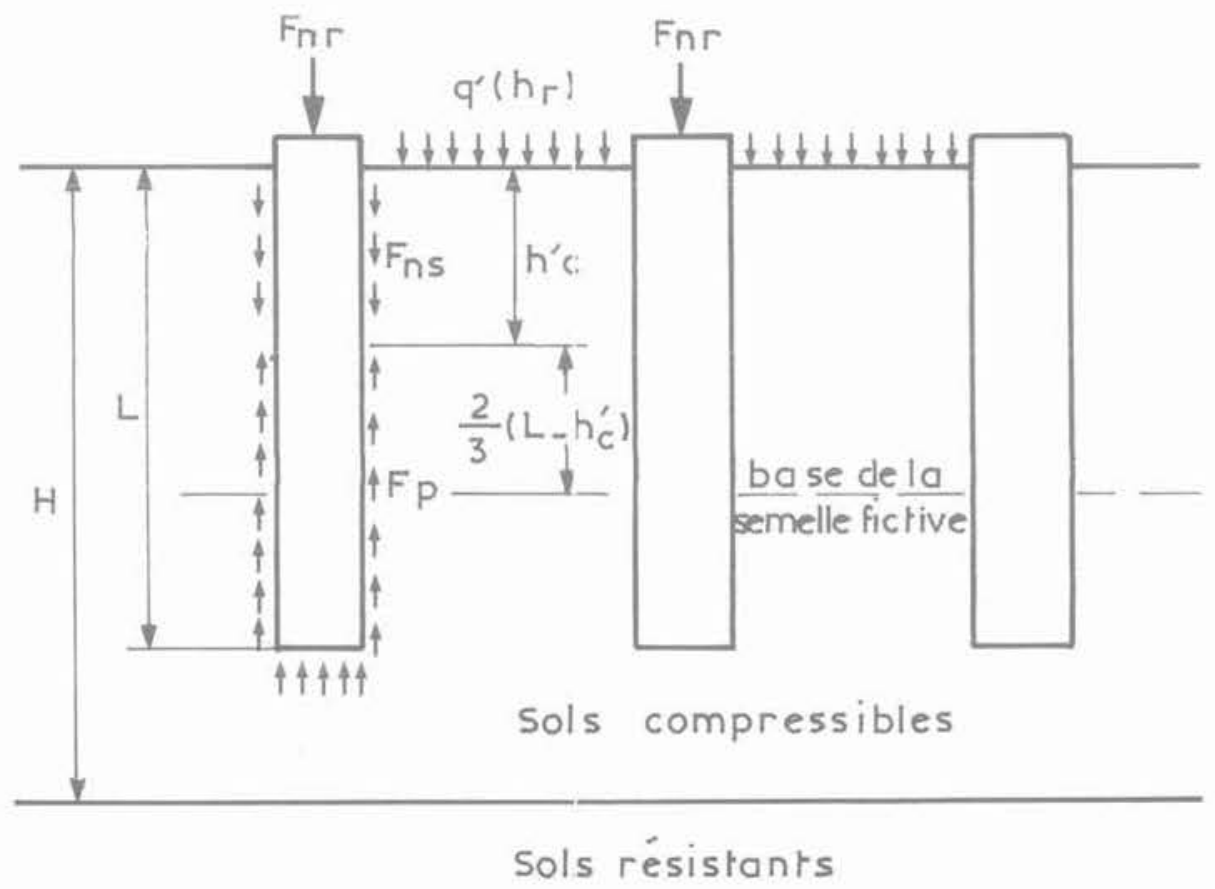

Fig. 11. - Inclusions arrêtées dans un sol médiocre. Fig. 11. - Piles tip in soft soil. 
Finalement, avec un réseau d'inclusions arrêtées dans le sol médiocre, toute la charge $\gamma_{t} \mathrm{~h}$ du remblai de tête est transmise au sol médiocre lui même. Il y a simplement une modification relativement complexe de cette transmission, qui conduit cependant à une réduction des tassements qui se produiraient sans le réseau.

Dans ce cas, d'ailleurs, la charge limite de la pointe de l'inclusion est mobilisée et il va de soi que la capacité de reprise en tête $\mathrm{F}_{\mathrm{nt}}$ est directement liée à la valeur de $Q_{p}$, charge limite en pointe de l'inclusion (voir 2.1.).

Il faut remarquer que, dans le cas du réseau arrêté sur le sol dur, une légère fraction de la charge en tête peut également, après transmission à l'inclusion (pour $\mathrm{z}<\mathrm{h}$ ), être retransmise au sol médiocre, par frottement positif, sur la hauteur $\mathrm{H}-\mathrm{h}$. Ce mécanisme a été volontairement négligé quant à sa contribution à un léger tassement supplémentaire, lequel est très faible.

\subsection{Adaptation de la géométrie des inclusions}

Partant d'une valeur $\mathrm{R}$ du rayon de l'inclusion en tête, on aboutit à un effort $\mathrm{Q}$, que l'inclusion doit supporter. On cherchera donc une dimension économique de celle-ci, pouvant amener à choisir un diamètre de la partie courante largement inférieur à $\mathrm{R}$, soit $\mathrm{R}^{\prime}<\mathrm{R}$ (fig. 12).

On est donc ramené à la géométrie schématisée cidessous (fig. 12).
Cette adaptation de la géométrie modifie néanmoins les conditions d'apparition du frottement négatif $F_{n s}$ sur le fût des inclusions. En effet, le rapport $\frac{b}{R}$ augmente sensiblement, l'importance de l'effet de groupe diminue, la surface du sol compressible, représenté par $\pi\left(b^{2}-R^{\prime 2}\right)$, augmentant devant une charge constante $\pi\left(b^{2}-R^{2}\right) q^{\prime}(h)$ qui lui est transmise. Il est donc nécessaire de vérifier quelles répercussions sur le tassement du sol, et sur la charge transmise aux inclusions, ce changement de géométrie entraîne.

On calculera donc le frottement négatif induit sur le réseau défini par $\frac{\mathrm{b}}{\mathrm{R}}$, sous l'effet d'une contrainte moyenne en tête du sol médiocre définie par

$$
\vec{q}^{\prime}\left(h_{r}\right)=\frac{b^{2}-R^{2}}{b^{2}-R^{12}} q^{\prime}\left(h_{r}\right)
$$

on a donc

$$
\overline{\mathrm{q}}^{\prime}\left(\mathrm{h}_{\mathrm{r}}\right)<\mathrm{q}^{\prime}\left(\mathrm{h}_{\mathrm{r}}\right)
$$

Il y a donc, puisque $\mathrm{R}^{\prime}<\mathrm{R}$, une réduction de l'effet de groupe au sein du sol; le terme $\mathrm{F}$ diminue, ce qui justifie a fortiori le passage de $\mathrm{R}$ à $\mathrm{R}^{\prime \prime}$. Par contre, bien sûr, les tassements sont en règle générale légèrement plus élevés, puisque l'efficacité du dispositif est moins bonne.

D'un point de vue pratique, sur chantier, on sera le plus souvent amené à prévoir, avant tout travaux, la mise en œuvre d'un remblai technique de travail d'épaisseur $h_{r 1}$, les inclusions étant mises en place

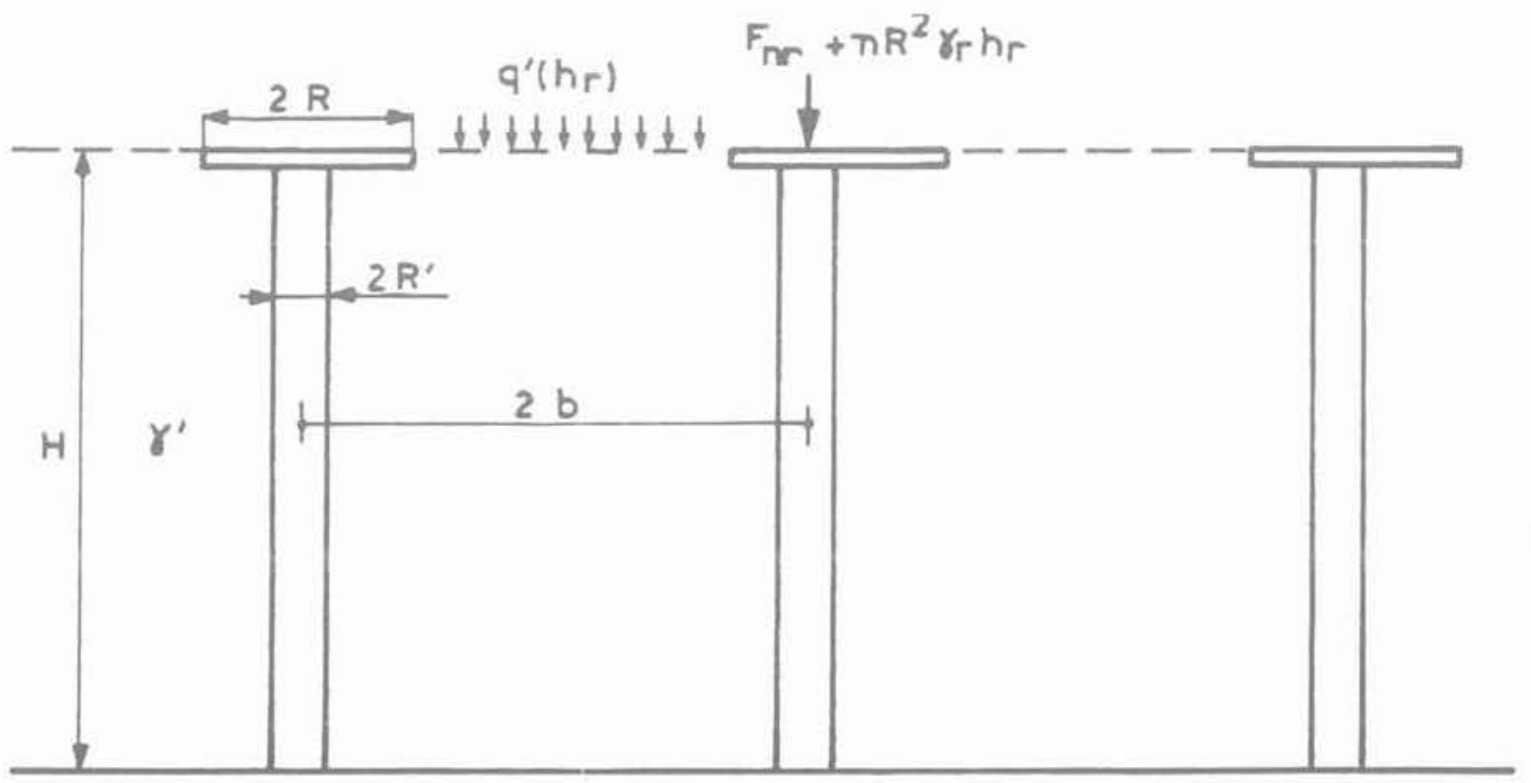

Fig. 12. - Inclusions surmontées d'une semelle.

Fig. 12. - Piles with cap. 
ultérieurement. On se trouve avec la géométrie représentée ci-dessous, après achèvement de l'ensemble du remblai à édifier, de hauteur totale $h_{r 1}+h_{r 2}$ (fig. 13).

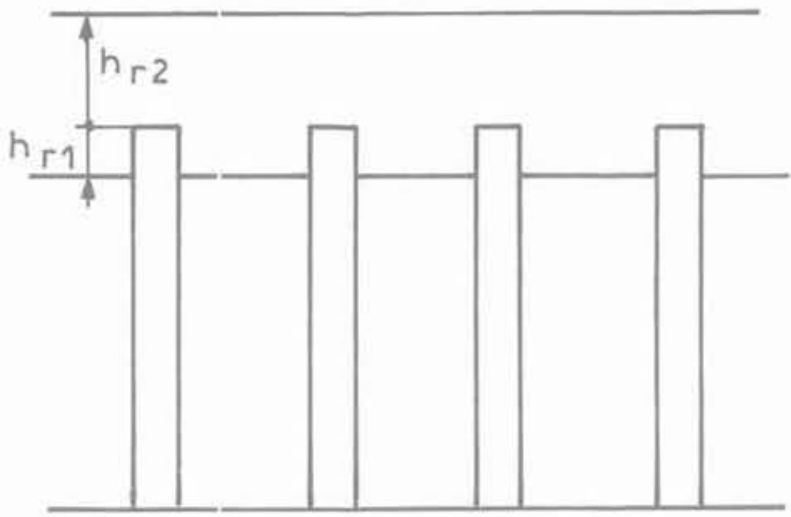

Fig. 13. - Réalisation pratique des inclusions. Fig. 13. - Pratical carrying out of piles.

Au niveau du calcul, cette disposition ne présente pas de difficulté particulière, puisque l'on peut distinguer deux couches de remblai $h_{r 2}$ et $h_{n}$.

\section{EXEMPLE D'APPLICATION}

La méthode précédente est appliquée au cas de la figure 14

$$
\begin{aligned}
& \text { remblai } \\
& \gamma_{r}=20 \mathrm{kN} / \mathrm{m}^{3} \\
& \mathrm{krg} \varphi_{r}=0,8 \\
& \begin{array}{l}
\text { sol compressible } \\
\gamma^{\prime}=7 \mathrm{kN} / \mathrm{m}^{3} \\
C_{c}=0,2 \\
1+e_{0} \\
\mathrm{krg} J=0,2
\end{array} \\
& \text { sol résistant,grave } \\
& \mathrm{Pl}=800 \mathrm{kPa}
\end{aligned}
$$

Fig. 14. - Exemple numérique. Fig. 14. - Numerical example.

Le remblai étant construit directement, le tassement calculé est, par méthode simplifiée:

$$
\Delta H=\frac{C_{c}}{1+e_{o}} \log \frac{\gamma_{r} h_{r}+\gamma^{\prime} \frac{H}{2}}{\gamma^{\prime} \frac{H}{2}} \cdot H=65 \mathrm{~cm}
$$

Le tassement prévisible (voir annexe) est de $77 \mathrm{~cm}$. On cherchera à réduire la contrainte sur le sol compressible à une valeur résiduelle $\mathrm{q}^{\prime}\left(\mathrm{h}_{\mathrm{r}}\right)=12 \mathrm{kPa}$.

Notons que cette contrainte, $\mathrm{q}^{\prime}\left(\mathrm{h}_{\mathrm{r}}\right)$, seule, ramène le tassement à $33 \mathrm{~cm}$.

\subsection{Calcul du réseau d'inclusions}

On choisira a priori $\mathrm{R}=0,15 \mathrm{~m}, \mathrm{q}^{\prime}(\mathrm{h})=\alpha \gamma_{f}, \mathrm{~h}_{\mathrm{r}}$ avec $\alpha=0,2$ donnant (abaque 2 et 3 ),

$$
\left\{m_{r}\left(0, \frac{b}{R}\right) \cdot h_{r}\right\} \text {, et } \frac{b^{2}}{R^{2}}=7,4 \text { d'où } \frac{d}{R}=2,4
$$

(maillage carré).

Dans le sol compressible, le coefficient d'accrochage $\lambda$ vaut 0,185 et $\frac{b}{R}=2,72$; pour ce couple $\lambda, \frac{\mathrm{b}}{\mathrm{R}}, \mathrm{m}\left(\lambda, \frac{\mathrm{b}}{\mathrm{R}}\right) \cdot \frac{\mathrm{R}}{\mathrm{K} \operatorname{tg} \delta}=0,36$ (abaque 3), d'où $\mathrm{m}\left(\lambda, \frac{\mathrm{b}}{\mathrm{R}}\right)=0,498$; puis $\mathrm{m}\left(\lambda, \frac{\mathrm{b}}{\mathrm{R}}\right) \times \frac{\mathrm{q}^{\prime}\left(\mathrm{h}_{\mathrm{c}}\right)}{\gamma^{\prime}}$ $=0,854$, et (abaque 4), $\mathrm{h}_{\mathrm{c}}=1,90 \mathrm{~m}$.

Il y a donc un point neutre à $1,90 \mathrm{~m}$, où la contrainte verticale dans le sol vaut

$$
\mathrm{q}(\mathrm{h}, \mathrm{R})=\gamma^{\prime} \mathrm{h}_{\mathrm{c}}=7 \times 1,95=13,28 \mathrm{kPa}
$$

La contrainte moyenne $\bar{q}(\mathrm{~h})$ dans le plan horizontal vaut donc, en vertu de (10):

$$
\bar{q}\left(h_{c}\right)=\gamma^{\prime} h_{c}+q^{\prime}\left(h_{r}\right)\left\{1-\frac{m\left(0, \frac{b}{R}\right)}{m\left(\lambda, \frac{b}{R}\right)}\right\}
$$

avec $\mathrm{m}(\mathrm{o}, \mathrm{b})=\frac{2 \pi \mathrm{R} \text { Ktg } \delta}{\pi\left(\mathrm{b}^{2}-\mathrm{R}^{2}\right)}=0,417$

d'où

$$
\overline{\mathrm{q}}\left(\mathrm{h}_{\mathrm{c}}\right)=13,28+12\left(1-\frac{0,417}{0,498}\right)=15,23 \mathrm{kPa}
$$


et, sur la hauteur $h$, la contrainte moyenne est, en vertu de (11): $\overline{\overline{\mathrm{q}}}=13,65 \mathrm{kPa}$.

Les tassements calculés pour ces états de contraintes sont finalement de $13 \mathrm{~cm}$ pour l'épaisseur $\mathrm{h}$, et de $2,2 \mathrm{~cm}$ pour la fraction restante $\mathrm{H}-h_{c}$; soit un tassement total de $15 \mathrm{~cm}$ environ.

En conclusion, pour un tassement prévisible de $77 \mathrm{~cm}$ sans dispositions particulières, la seule prise en compte du développement de l'effet de voûte en tête des inclusions amène cette valeur à $33 \mathrm{~cm}$. Ce premier effet réducteur. complété par l'apparition du frottement négatif sur le réseau d'inclusions, conduit en définitive à un tassement de $15 \mathrm{~cm}$ environ. Le tassement obtenu est donc cinq fois plus faible.

\subsection{Efforts dans les inclusions}

L'effort transmis en tête, vaut

$$
\begin{aligned}
F_{n x}+\pi R^{2} \gamma_{r} h_{r} & =\pi R^{2} \gamma_{r} h_{r} \\
& \left\{\frac{b^{2}}{R^{2}}(1-\alpha)-\alpha\right\}=25,9 \mathrm{kN}
\end{aligned}
$$

Par frottement négatif, apparait le long du fût un effort

$$
F_{n, s}=\frac{2 \pi R K \operatorname{tg} \delta}{m\left(\lambda, \frac{b}{R}\right)} q^{\prime}\left(h_{r}\right)=4,5 \mathrm{kN}
$$

d'où

$$
Q_{1}=F_{n, r}+F_{n s}+\pi R^{2} \gamma_{r} h_{r}=30,4 \mathrm{kN}
$$

Donc, pour un pilot de type battu posé au toit de la grave, de pression limite $P_{1}-P_{0}=800 \mathrm{kPa}$, avec un facteur de portance de 4, la capacité portante en pointe $Q_{p l}$ vaut $(0,15)^{2} \times 4 \times 800=225 \mathrm{kN}$; ce pilot peut donc encaisser, sans déformation appréciable, $120 \mathrm{kN}$, bien supérieurs aux $30,4 \mathrm{kN}$ à reprendre. La section peut donc être réduite, dans la mesure où les contraintes de compression sont admissibles. En admettant $3000 \mathrm{kPa}$, on peut envisager de réduire de moitié le rayon $\mathrm{R}$ initial du pilot et de le ramener à $\mathrm{R}^{\prime}$ $=7,5 \mathrm{~cm}$.

Examinons la répercussion de cet abaissement de $\mathrm{R}$ à $\mathrm{R}^{\prime}$ sur le tassement. On a en effet:

$$
\frac{\mathrm{b}}{\mathrm{R}^{\prime}}=2 \frac{\mathrm{b}}{\mathrm{R}}=5,44
$$

Dans ces conditions, on trouve successivement $\mathrm{m}\left(\mathrm{o}, \frac{\mathrm{b}}{\mathrm{R}}\right)=0,186, \mathrm{~m}\left(\lambda, \frac{\mathrm{b}}{\mathrm{R}}\right)=0,312, \mathrm{~h}_{\mathrm{c}}=2,53 \mathrm{~m}$, $\overline{\mathrm{q}}(\mathrm{h})=22,54 \mathrm{kPa}$ et $\overline{\overline{\mathrm{q}}}=17,47 \mathrm{kPa}$.
Le tassement final calculé est de $21 \mathrm{~cm}$ avec $17,3 \mathrm{~cm}$ pour l'épaisseur $h_{c}$ et $3,7 \mathrm{~cm}$ pour la partie inférieure $\mathrm{H}-\mathrm{h}_{\mathrm{c}}$.

Cette valeur est donc supérieure à celle obtenue avec les inclusions de rayon $\mathrm{R}$ initialement choisi.

Quant aux efforts dans le pilot, ils sont réduits dans des proportions minimes, sachant qu'en tête il n'y a pas de modification; $F_{n s}$ est donné par

$$
F_{n s}=\frac{2 \pi R^{\prime} K \operatorname{tg} \delta}{m\left(\lambda, \frac{b}{R^{\prime}}\right)} \text { ou }{ }^{\prime} R^{\prime}=0,075 m
$$

au lieu de $R=0,15 \mathrm{~m}$, et $\mathrm{m}\left(\lambda, \frac{\mathrm{b}}{\mathrm{R}^{\prime}}\right)=0,312$ au

lieu de 0,498 précédemment, d'où $F_{m}=3,6 \mathrm{kN}$, soit $\mathrm{Q}_{t}=29,5 \mathrm{kN}$.

Remarque. - Si l'on s'avisait d'arrêter les pilots avant le toit de grave, à $4 \mathrm{~m}$ par exemple, on peut voir rapidement, sans calculs approfondis, que l'efficacité d'une telle disposition serait extrêmement faible et, bien sûr, sans aucun intérêt.

\section{DONNÉES EXPÉRIMENTALES}

L'examen détaillé de la littérature technique n'a porté que sur la construction de remblais sur sol amélioré par inclusions.

\subsection{Etude expérimentale de KORFIATIS (1984)}

Une première expérimentation intéressante, car elle permet une confrontation avec la méthode proposée, est celle décrite par KORFIATIS (1984).

On a instrumenté trois aires d'essai, comportant chacune un remblai de $3,70 \mathrm{~m}$ de hauteur reposant sur $3,90 \mathrm{~m}$ de sols compressibles tourbeux, organiques, saturés. Deux zones comportant des minipieux de bois de diamètre $0,35 \mathrm{~m}$, disposés selon des mailles carrées de $1,22 \mathrm{~m}$ et $1,52 \mathrm{~m}$ de côté $(2 \mathrm{~d})$ respectivement, et une zone sans minipieux, ont été suivies pendant plusieurs mois, au niveau des tassements de surface. Un géotextile Mirafi 500 X garnissait la base du remblai.

La figure 15 donne quelques détails sur l'expérimentation.

Au niveau des tassements, les valeurs mesurées sont données dans le tableau 1. Au bout de 190 jours, l'évolution est beaucoup plus lente pour le maillage le plus serré $(0,9 \mathrm{~mm} /$ jour, $0,5 \mathrm{~mm} /$ jour et $0,2 \mathrm{~mm} /$ jour, respectivement). 


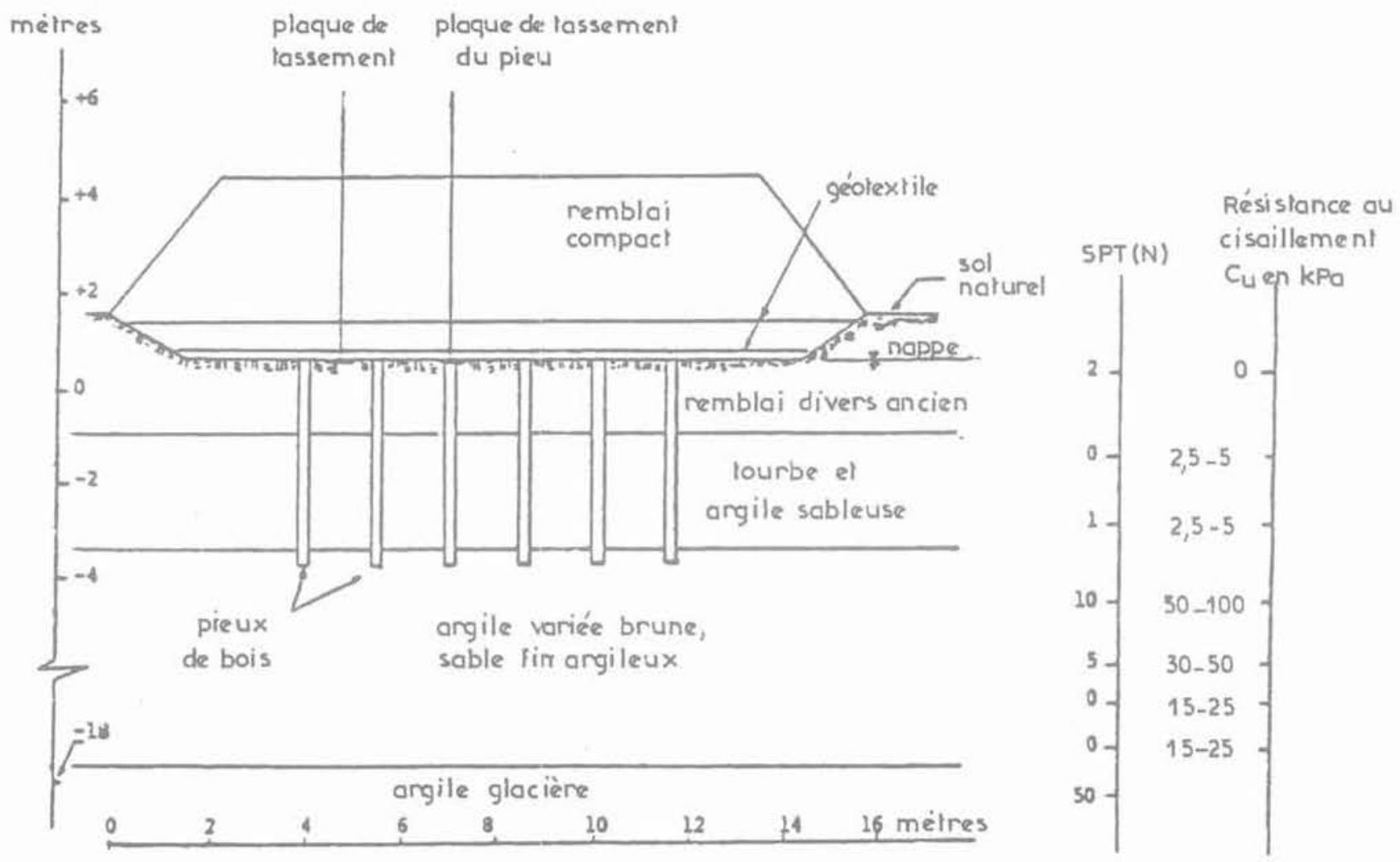

distance horizontale

Fig. 15. - Expérimentation d'un remblai aux U.S.A. (d'après Korfiatis).

Fig. 15. - Fill experimentation in USA (after Korfiatis).

Tableau 1. - Tassements mesures.

Table 1. - Measured settlements

\begin{tabular}{|l|c|c|c|}
\hline \multicolumn{1}{|c|}{ Remblai } & Référence & Maille de $1,22 \times 1,22 \mathrm{~m}$ & Maille de $1,52 \times 1,52 \mathrm{~m}$ \\
\hline $\begin{array}{l}\text { Tassement } \\
\text { primaire } \\
(60 \text { à } 80 \text { jours })\end{array}$ & $39,6 \mathrm{~cm}$ & $18,3 \mathrm{~cm}$ & $30,5 \mathrm{~cm}$ \\
\hline $\begin{array}{l}\text { Tassement } \\
\text { à } 190 \text { jours }\end{array}$ & $61 \mathrm{~cm}$ & $21,3 \mathrm{~cm}$ & $39,6 \mathrm{~cm}$ \\
\hline
\end{tabular}

Afin d'interpréter ces résultats, certaines hypothèses ont dû être faites pour remplacer les données manquantes, On a choisi pour le remblai un poids volumique $\gamma$, de $20 \mathrm{kN} / \mathrm{m}^{3}$ et des coefficients $\mathrm{Ktg}$, de 0,8 et 1 , successivement. Pour le sol compressible, on a adopté un poids volumique déjaugé $\gamma^{\prime}$ de $6 \mathrm{kN} / \mathrm{m}^{3}$, et des coefficien,ts Ktgò de 0,15 (coefficient d'accrochage $\lambda=$ $0,235)$ et $\mathrm{Ktg} \delta=0,25(\lambda=0,135)$.

Les maillages ( $2 \mathrm{~d}=1,22$ et 1,52 $\mathrm{m}$, ce qui correspond à $\frac{b}{R}=3,93$ et 4,90 ), sont donc relativement lâches.

$\mathrm{Ne}$ connaissant pas les caractéristiques de compressibilité des sols, on a assimilé la couche de $3,90 \mathrm{~m}$ d'épaisseur à un matériau homogène.
Pour le remblai test (sans minipieux) les $61 \mathrm{~cm}$ de tassement observés en 190 jours (tassements primaire et secondaire) ont permis de calculer un coefficient $\frac{C_{c}}{1+e_{0}}$ équivalent de 0,157 ; si lon se limite au tassement primaire, $(39,6 \mathrm{~cm})$, on obtient une valeur de $\frac{C_{c}}{1+e_{o}}$ de 0,10 .

Sur la base de ces données, on a calculé par la méthode exposée les tassements avec inclusions, (tableau 2). 
Tableau 2. - Tassements calculés (cm)

Table 2. - Calculated settlements

\begin{tabular}{|c|c|c|c|c|c|c|c|c|}
\hline Ktg甲 & \multicolumn{4}{|c|}{0,8} & \multicolumn{4}{|c|}{1} \\
\hline$\gamma^{\prime}$ Sol compressible & \multicolumn{8}{|c|}{$6 \mathrm{kN} / \mathrm{m}^{3}$} \\
\hline Ktgò Sol compressible & \multicolumn{2}{|c|}{0,15} & \multicolumn{2}{|c|}{0,25} & \multicolumn{2}{|c|}{0,15} & \multicolumn{2}{|c|}{0,25} \\
\hline $\mathrm{b} / \mathrm{R}$ & 3,93 & 4,90 & 3,93 & 4.90 & 3,93 & 4,90 & 3,93 & 4,90 \\
\hline $\begin{array}{l}\text { W calculé avec } \\
\qquad \frac{C_{c}}{1+e_{o}}=0,157\end{array}$ & 34,2 & 42,3 & 29,5 & 39,8 & 30,7 & 39,1 & 27 & 37 \\
\hline $\begin{array}{l}\text { W calcul avec } \\
\frac{C_{c}}{1+e_{0}}=0,10\end{array}$ & 22,2 & 27,5 & 19,2 & 25,9 & 20 & 25,4 & 17,6 & 24,1 \\
\hline
\end{tabular}

La comparaison des calculs et des mesures ne permet pas de choisir la bonne valeur des paramètres. Mais les calculs, comme les mesures, montrent une réduction appréciable des tassements du sol compressible du fait du réseau d'inclusions, avec des ordres de grandeur raisonnable compte tenu de toutes les incertitudes.

Il faut noter que les pointes des pieux ont poinçonné les marnes sous-jacentes, puisque les pieux ont tassé de 3 et $4,5 \mathrm{~cm}$, respectivement. Ces tassements sont tout à fait logiques puisque dans le cas du réseau le plus lâche $\left(\frac{b}{R}=4,90\right)$ et pour $\operatorname{Ktg}_{\varphi_{x}}=0,8$ et

Ktg $\delta=0,25$, la charge $Q$ transmise à un minipieu atteint $119 \mathrm{kN}$; la charge limite en pointe est de l'ordre de $90 \mathrm{kN}$, d'après les caractéristiques géotechniques du sol. Il y a donc bien poinçonnement des pointes et, de ce fait, réduction du frottement négatif $F_{n s}$; l'efficacité du groupe est donc moins bonne que si les pieux reposaient sur un sol plus résistant.

D'autre part, la présence d'un géotextile au dessus des têtes de pieux donne globalement au remblai une cohésion équivalente, ici probablement faible, mais dont Pinfluence est bénéfique pour les tassements et croît rapidement avec le resserrement du maillage (voir an nexe II).

\subsection{Chantiers en Ecosse \\ (REID et BUCHANAN, 1983)}

Plusieurs remblais d'accès, à des ouvrages d'art, sur sols compressibles ont été édifiés sur des réseaux de pieux portant des semelles circulaires préfabriquées. Deux lits de TERRAM (membrane géotextile) renforcé de lanières, ont été disposés au-dessus de ces semelles pour favoriser l'effet de voûte.

Les pieux sont analogues à ceux utilisés pour l'ouvrage d'art; leur espacement est modulé avec la distance à la culée, de même que leur longueur, de sorte qu'on aménage une zone de transition progressive, où les tassements passent d'une valeur faible près de la culée à la valeur plus forte du tassement du sol sous le remblai en l'absence d'inclusions.

La capacité portante des pieux est aménagée de sorte qu'une sécurité de 2 , près de la culée, conduise à une efficacité maximale des inclusions; ioin de la culée, la sécurité est plus faible.

La figure 16 montre clairement le dispositif mis en œuvre pour l'un des ouvrages d'art ainsi conçus.

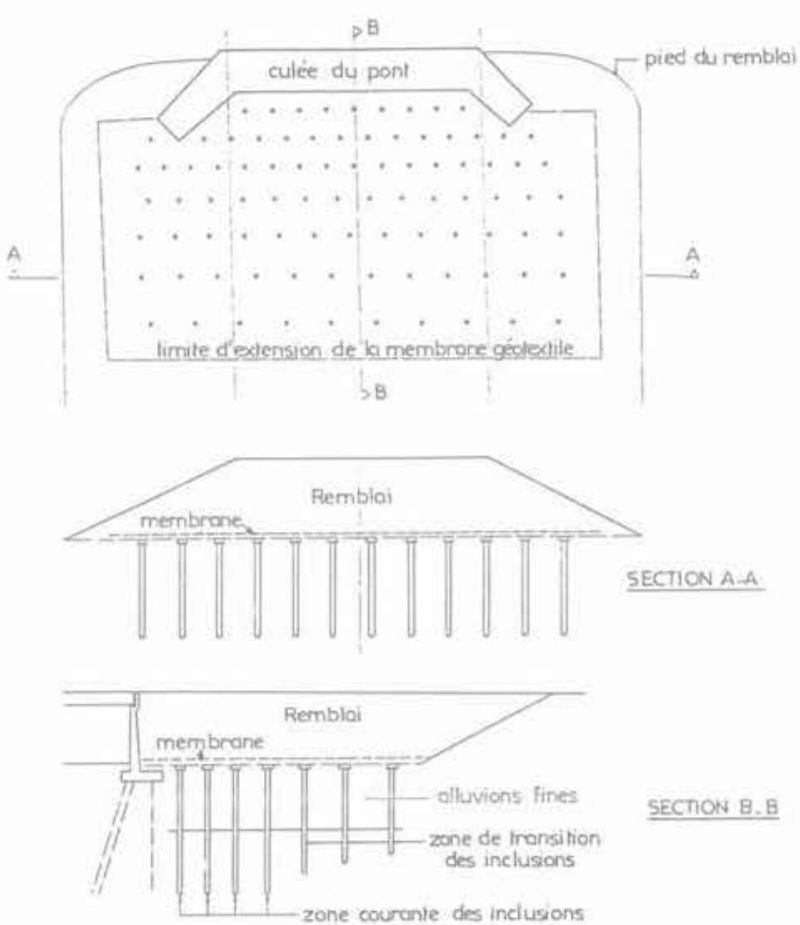

Fig. 16. - Principe des remblais d'accès à des ouvrages d'art en Ecosse (Reid et Buchanan).

Fig. 16. - Conception of embankments near bridges in scotland

(after Reid and Buchanan) 
Une instrumentation poussée a permis de suive les tassements, les pressions interstitielles, la charge en tête des inclusions et les déplacements horizontaux, à la fois dans la zone améliorée et hors de celle-ci.

Il apparait sans aucune ambiguité que, dans la zone traitée, un pourcentage très important de la charge de remblai passe dans l'inclusion (environ $80 \%$ ) ; les pressions interstitielles développées restent extrêmement faibles (quelques pourcents) et les déplacements horizontaux du sol sont réduits dans des proportions considérables (environ $1 \mathrm{~cm}$ contre $14 \mathrm{~cm}$ ).

A partir des quelques données de la publication, la méthode proposée en 3 a été appliquée.

On a retenu les chiffres suivants pour la zone où le réseau des pieux est le plus serré, près de la culée:

$\frac{b^{2}}{R^{2}}=7,70$ :

$\mathrm{R}=0,6 \mathrm{~m} ; \gamma_{t} \mathrm{~h}_{t}=130 \mathrm{kPa} ; \gamma_{\mathrm{r}}=13 \mathrm{kN} / \mathrm{m}^{3}$.

et l'on a choisi $\mathrm{Ktg}_{\varphi_{\mathrm{r}}}=0,8$ et 1 , respectivement.

Dans ces conditions, on a respectivement :

$\mathrm{m}\left(\lambda, \frac{\mathrm{b}}{\mathrm{R}}\right)=0,4$ et 0,5 et $\mathrm{q}^{\prime}\left(\mathrm{h}_{r}\right)=31,9$ et $25,8 \mathrm{kPa}$;

Ce qui conduit, en tête des inclusions, à des efforts de 860 et $905 \mathrm{kN}$, à comparer aux $900 \mathrm{kN}$ mesurés sur le site.

\subsection{Techniques utilisées en Suède (BROMS, 1979)}

BROMS (1979) décrit les procédés utilisés en Suède pour construire sur pieux certains remblais d'accès à des culées d'ouvrages d'art. Les pieux reposent sur un sol suffisamment résistant et sont coiffés d'une semelle individuelle ( a pile cap $»$ ) assurant un taux de couverture variable.

En particulier, le code routier suêdois propose pour le dimensionnement l'abaque de la figure 17, qui donne le maillage nécessaire pour reprendre $80 \%$ du poids du remblai $(\beta=0,2)$. La méthode suédoise étant d'origine expérimentale, la courbe est comparée à celles données par la méthode proposée dans cet article, pour $\mathrm{Ktg}_{\varphi_{t}}=0,5-0,75$ et $\mathrm{I}$.

\subsection{Techniques utilisées en Finlande (RATHMAYER, 1975)}

RATHMAYER (1975) décrit trois remblais instrumentés, où la hauteur, la nature du matériau et le taux de couverture des semelles sur pieux varient, et pour lesquels plus de $80 \%$ des charges sont transmises aux inclusions. La méthode utilisée, comme en Suède, permet d'édifier les remblais immédiatement après la mise en ceuvre des inclusions.

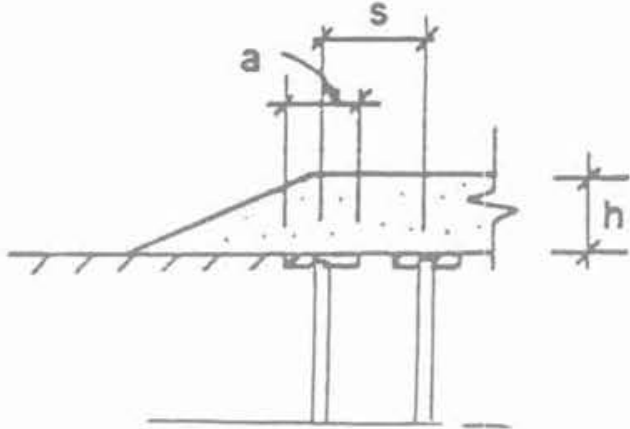

Fig 170

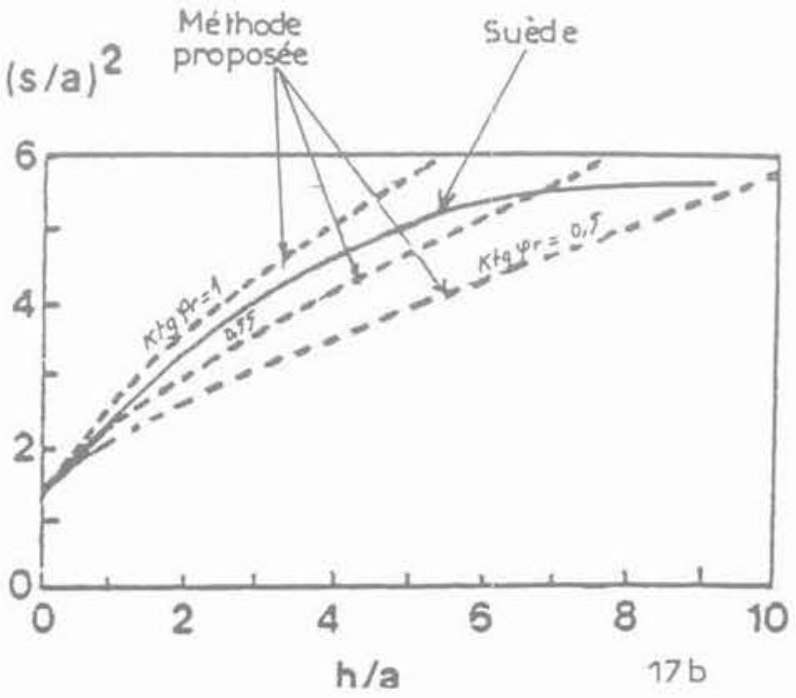

Fig. 17. - Abaque de dimensionnement des réseaux de pieux (d'après Broms).

Fig. 17 - Graph for piles network design (after Broms)

Dans les pays scandinaves le bois est un matériau bon marché et cette tehnique s'avère économiquement intéressante. Le code router finnois donne une méthode de dimensionnement d'origine expérimentale.

RATHMAYER (1975) donne par ailleurs le tableau 3 ci-dessous, qui fait bien apparaître, quand la hauteur du remblai croît, un maillage de plus en plus lâche des têtes d'inclusions, qui doivent reprendre un pourcentage très élevé des charges de remblai ( $80 \%$ au minimum).

Tableau 3 - Taux de couverture en $\%$ des inclusions Table 3. - Covering ratio of piles

\begin{tabular}{|c|c|c|}
\hline $\begin{array}{c}\text { Hauteur } \\
\text { du remblai } \\
\text { (mètres) }\end{array}$ & $\begin{array}{c}\text { Remblai } \\
\text { de roche } \\
\text { concassée }\end{array}$ & $\begin{array}{c}\text { Remblai } \\
\text { en grave }\end{array}$ \\
\hline $1,5-2$ & $50-70$ & $>70$ \\
$2-2,5$ & $40-50$ & $55-70$ \\
$2,5-3$ & $30-40$ & $45-55$ \\
$3-3,5$ & $30-40$ & $40-45$ \\
$3,5-4$ & $>30$ & $>40$ \\
\hline
\end{tabular}




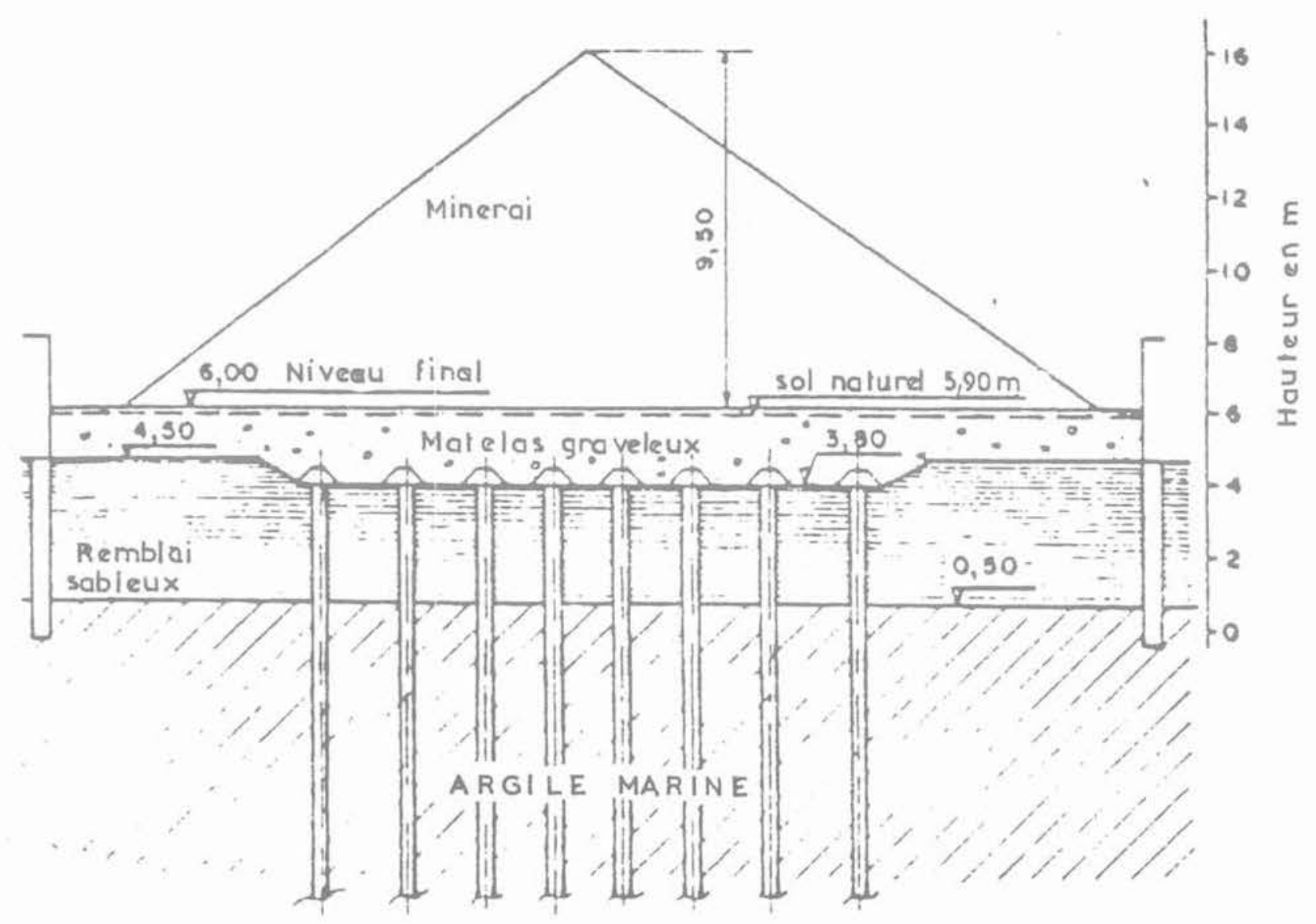

Fig. 18. - Coupe de l'aire de stockage sur pieux (Souto et al.).

Fig. 18. - Iron stockage on piles (after Souto and al.).

\subsection{Aires de stockage de minerai au Brésil (SOUTO et al., 1983)}

La conception d'aires de stockage de minerai de fer très lourd à São Paulo, Brésil, décrite par SOUTO et al. (1983), relève du même principe.

Le stockage de près de 10 mètres de ce matériau se fait sur 25 mètres d'argile marine (fig. 18); les solutions rigides traditionnelles ont été abandonnées au profit de solutions souples, mettant en jeu les effets de voûte dans le matériau et consistant à transmettre les efforts dans des pieux par l'intermédiaire d'un remblai très frottant et de semelles indépendantes coiffant la tête des pieux. Le calcul par la méthode des éléments finis prévoyait la reprise par les pieux de $85 \%$ de la charge, avec des tassernents de $3 \mathrm{~cm}$.

Les mesures réalisées ont donné des déformations verticales de $3.4 \mathrm{~cm}$, avec $80 \%$ de la charge passant dans les fondations. Il faut signaler que le tassement prévu (sans amélioration du sol) par les mêmes méthodes de calcul aux éléments finis dépassait 2 mètres.

Cette solution a conduit à $40 \%$ d'économie, par comparaison à une méthode plus traditionnelle.

\subsection{Etudes sur modèles réduits (TING et al., 1983)}

Enfin, nous citerons les études sur modèles réduits de TING et al. (1983), en Malaisie, qui ont fait varier les hauteurs et densités des charges de remblai et l'espacement des inclusions de $38 \mathrm{~mm}$ de diamètre. Les résultats des mesures sont comparés à des calculs en êléments finis (fig. 19).

Les auteurs concluent que la charge transmise aux inclusions équivaut au poids d'un prisme de remblai, appelé «zone de rupture » et dont le diamètre est 3 à 4 fois celui des inclusions (fig. 19); le résultat de ces essais montre bien qu'il y a une transmission importante des efforts dans les inclusions.

\section{CONCLUSION}

La construction des remblais sur sols compressibles conduit très souvent à une surconsommation importante de matériau par suite des tassements importants dont les sols médiocres sont le siège. Des problèmes 


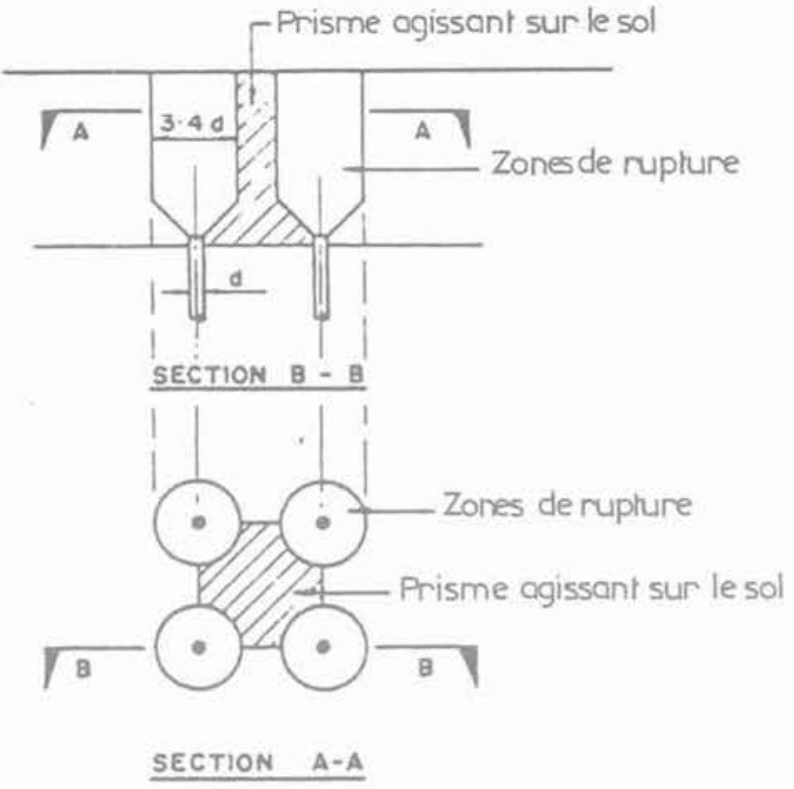

Fig. 19. - Essais sur modèles réduits (Ting et al.). Fig. 19. - Tests with laboratory model (after Ting and al.).

de délais d'exécution s'ajoutent généralement aux précédents, liés à la stabilité.

Une méthode d'amélioration des sols compressibles consiste à les renforcer par des inclusions verticales rigides. Une méthode de dimensionnement de ces réseaux est décrite dans ce rapport.

Comme le montre l'expérience accumulée à l'étranger, le renforcement par pieux des sols compressibles sous les remblais permet:

- de diminuer les tassements dans des proportions considérables :

- de construire immédiatement les remblais,

- de limiter les mouvements latéraux.

— et d'assurer la stabilité des remblais à court terme.

Cette étude et la méthode proposée ont été limitées au seul domaine de la construction de remblais.

Les cas des radiers et des semelles seront traités ultérieurement.

\section{Annexe 1: remarque sur le calcul du tassement d'une épaisseur de sol compressible}

Cette annexe examine les répercussions entrainées sur les valeurs de tassement calculées par les méthodes simplificatrices couramment utilisées. Quoique connues, il nous a paru utile de les rappeler. On raisonne ici en sol homogène, normalement consolidé, et répondant au modèle de TERZAGHI.

On considère une couche d'épaisseur $\mathrm{H}$. A la cote $\mathrm{z}=\mathrm{o}$, la contraite effective initiale, avant chargement sous $\mathrm{q}$, est $\sigma_{\mathrm{w}}^{\prime}$; le sol a un poids volumique déjaugé $\gamma^{\prime}$.

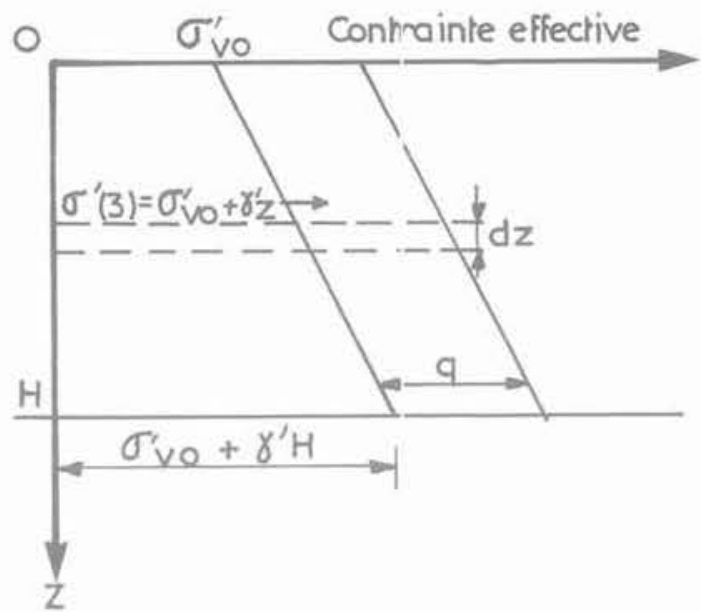

Le tassement de la couche élémentaire dz est égal à :

$$
\frac{d w}{d z}=0,435 \frac{C_{c}}{1+1} \ln \frac{q+\sigma_{w_{0}}^{\prime}+\gamma^{\prime} z}{\sigma_{v_{0}}^{\prime}+\gamma^{\prime} z}
$$

On pose $0,435 \frac{C_{c}}{1+10}$.

On en déduit la compression de la couche d'épaisseur $\mathrm{H}$ :

$$
w=k \int_{0}^{+1} \ln \left(1+\frac{q}{\sigma_{\psi_{0}}^{\prime}+\gamma^{\prime} z}\right) d z
$$

laquelle s'écrit tous calculs faits:

$$
\begin{aligned}
\frac{w}{k}= & \frac{q}{\gamma^{\prime}} \ln \left(1+\frac{\gamma^{\prime} H}{\sigma_{v_{0}}^{\prime}+q}\right) \\
+ & \left(H+\frac{\sigma_{v 0}^{\prime}}{\gamma^{\prime}}\right) \ln \left(1+\frac{q}{\sigma_{v 0}^{\prime}+\gamma^{\prime} H}\right) \\
& \left.-\frac{\sigma_{v_{0}^{\prime}}^{\prime} \ln \left(1+\frac{q}{\gamma^{\prime}}\right)}{\sigma_{v_{0}}^{\prime}}\right)
\end{aligned}
$$

Cette valeur est à comparer à la valeur simplifiée couramment employée:

$$
\frac{w}{\mathrm{k}}=\ln \frac{2 \mathrm{q}+2 \sigma_{v_{0}}^{\prime}+\gamma^{\prime} \mathrm{H}}{2 \sigma_{v_{0}}^{\prime}+\gamma^{\prime} \mathrm{H}}
$$

qui considère la valeur de la contrainte effective au milieu de la couche $\mathrm{H}$.

En particulier, si $\sigma_{{ }_{0}}^{\prime}=o$, le tassement $\mathrm{W}$ en tête est égal à : 


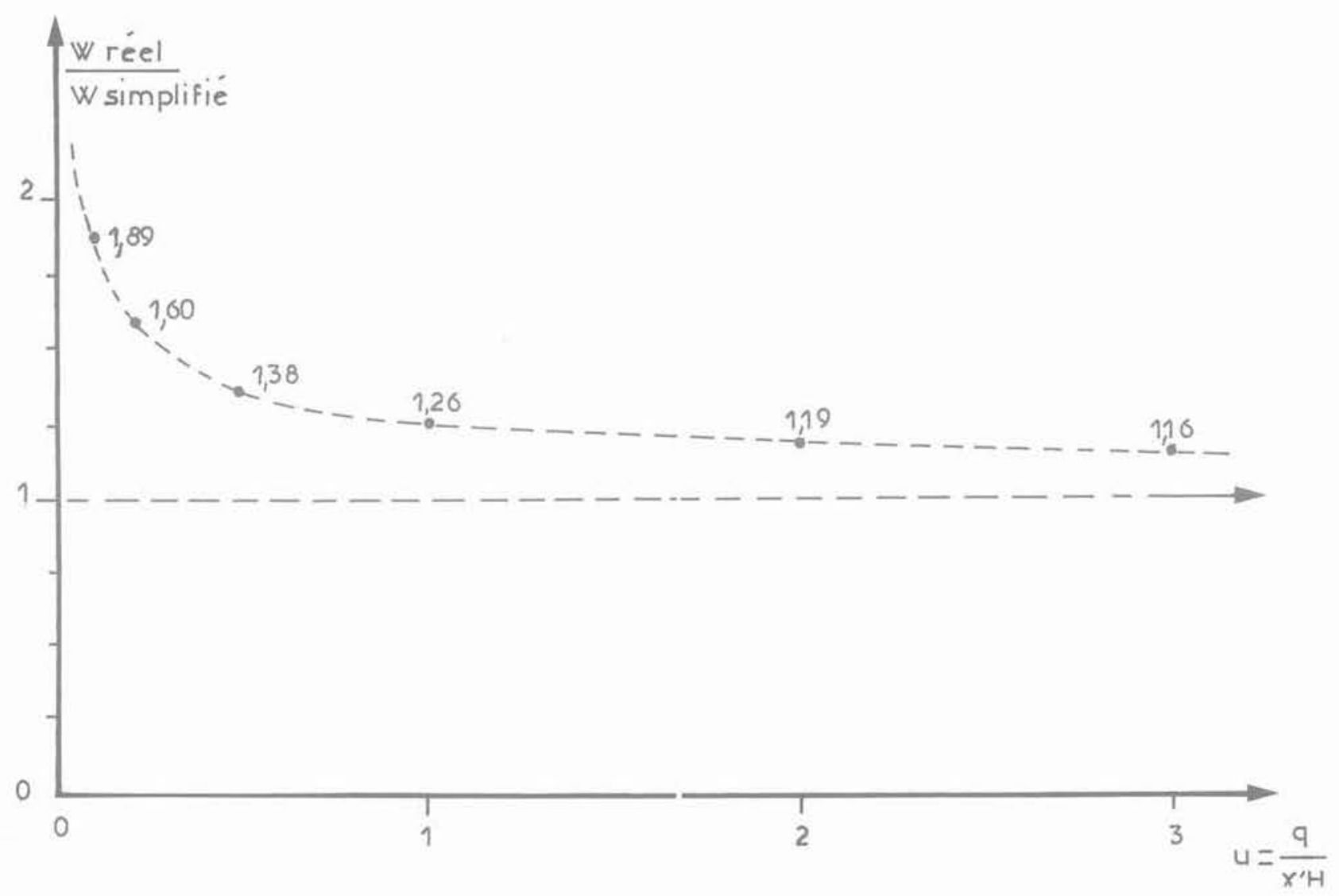

Fig. 20. - Rapport des tassements réels et simplifiés (cas où $\sigma_{v_{0}}^{\prime}=0$ ).

Fig. 20 - Ratio of exact and simplified calculated settlements.

$$
\begin{aligned}
& \frac{W_{\text {rie }}}{k}=\frac{q}{\gamma^{\prime} H} \ln \left\{1+\frac{\gamma^{\prime} H}{q}\right\} \\
& +\ln \left\{1+\frac{\mathrm{q}}{\gamma^{\prime} \mathrm{H}}\right\} \\
& \text { et } \frac{W_{\text {simp }}}{k}=\ln \left\{1+\frac{2 q}{\gamma^{\prime} H}\right\}
\end{aligned}
$$

Le rapport $\frac{W_{\text {reit }}}{W_{\text {simpl- }}}$ varie avec $\frac{q}{\gamma^{\prime} H}$, suivant le graphe cidessus (fig. 20). Pour $u=\frac{q}{\gamma^{\prime} \mathrm{H}}$ très grand, ce rapport vaut $\frac{1+\ln u}{\ln 2 u} ;$ quant $\mathrm{u}$ tend vers $\mathrm{O}$, il tend vers $\frac{1}{2}$ $(1-\ln u)$.

On tiendra compte de cette remarque importante, dans les calculs auxquels il est procédé dans cette étude.

\section{Annexe 2: influence de la cohésion du remblai}

Si l'on caractérise le remblai par $\varphi_{r}$ et $C_{r}$, on montre sans difficulté en admettant entre les inclusions, au sein du remblai, une contrainte $\mathrm{q}^{\prime}(\mathrm{z})$ uniforme (du fait d'un accrochage $\lambda=0$, très intense) et un déplacement de sol suffisant, que

$$
q^{\prime}(z)=\frac{1}{m\left(0, \frac{b}{R}\right)}
$$

$$
\left\{\gamma_{r}-\frac{m\left(0, \frac{b}{R}\right) C_{r}}{\operatorname{Ktg}_{\varphi}}\right\} \cdot\left\{1-e^{-m\left(0, \frac{b}{R}\right)}\right\}
$$

$$
m\left(o, \frac{b}{R}\right) C_{r}
$$

Le terme $\gamma_{r}-\frac{(\mathrm{R})}{\mathrm{K} \varphi_{\varphi_{\mathrm{r}}}}$ fait donc apparaître une cohésion critique dépendant du maillage et telle que, pour cette valeur, $\mathrm{q}^{\prime}(\mathrm{z})=0$; pour $\mathrm{C}_{\mathrm{t}}>\mathrm{C}_{\mathrm{r}}$, it il ne passe donc pas d'effort dans le sol compressible, tout l'effort passant dans les inclusions; pour $\mathrm{C}_{r}<\mathrm{C}_{\mathrm{r} \text { cin }}$, comme 


$$
\begin{aligned}
\mathrm{F}_{\mathrm{n} x}= & 2 \pi \mathrm{R} \int_{0}^{\mathrm{h}_{\mathrm{t}}} \tau(\mathrm{z}) \mathrm{dz}= \\
& 2 \pi \mathrm{R} \int_{0}^{\mathrm{h}_{\mathrm{t}}}\left\{\mathrm{C}_{r}+\operatorname{Ktg}_{\mathrm{r}} \mathrm{q}^{\prime}(\mathrm{z})\right\} \mathrm{dz}
\end{aligned}
$$

on montre que

$$
F_{n, s}=\frac{2 \pi R \operatorname{Ktg}_{\varphi_{T}}}{m\left(0, \frac{b}{R}\right)}\left\{\gamma_{\tau} h_{r}-q^{\prime}\left(h_{r}\right)\right\}
$$

qui peut s'écrire

$$
\begin{aligned}
F_{n s}=F_{n, x}\left(\varphi_{r}\right)+\frac{2 \pi R C_{r}}{m\left(0, \frac{b}{R}\right)} & \left\{1-e^{-m\left(0, \frac{b}{r}\right) h_{r}}\right\}
\end{aligned}
$$

où $\mathrm{F}_{0}\left(\varphi_{-}\right)$est le terme correspondant à $\mathrm{C}=0$; la cohésion $\mathrm{C}_{r}$ ajoute à l'effort $\mathrm{F}_{n}$, dû au frottement $\phi_{r}$, un terme complémentaire, directement lié au maillage.

En particulier, on peut, dans le cas d'un remblai renforcé (géotextiles ou tout autre procédé), introduire une cohésion équivalente, qui sera identifiée à un terme $C_{r}$.

\section{BIBLIOGRAPHIE}

1. ABBS A.F. (1984), Le renforcement par pieux des sols de fondation d'un réservoir de pétrole. Comptes rendus, Colloque International sur le renforcement en place des sols et des roches, Paris, Presses de l'E.N.P.C., pp. 13-18.

2. BROMS B. (1979), Problems and solutions to construction in soft clay. Proceedings, 6th Asian Regional Conférence on Soil Mechanics and Foundation Engineering, Singapore, vol. 2, pp. 28-30.
3. COMBARIEU O. (1974), Effet d'accrochage et méthode d'évaluation du frottement négatif. Bulletin de liaison des Laboratoires des Ponts et Chaussées, nं 71, pp. 93-107.

4. COMBARIEU O. (1985), Frottement négatif sur les pieux. Laboratoire Central des Ponts et Chaussées, rapport de recherche L.P.C., $\mathrm{n}^{\circ} 136,152 \mathrm{p}$.

5. GIGAN J.P. (1975), Consolidation d'un sol de fondation par pilots. Bulletin de liaison des L.P.C., $\mathrm{n}^{\circ} 78$, pp 12-16.

6. KORFIATIS G.P. (1984), Field testing of short pile systems for floor support. Stevens Institute of Technology USA. Comptes rendus, Colloque International, "renforcement en place des sols et des roches , Paris, Octobre 1984, Presses de l'E.N.P.C., pp. $71-75$.

7. MENARD L. (1969), Ancrages à géométrie variable. Notice D/93/69, Société Louis MENARD, $8 \mathrm{p}$.

8. NGUYEN THANH LONG, MORBOIS A. (1984), Etude du procédé ACTIMUR. Laboratoire Central des Ponts et Chaussées, rapport des Laboratoires série GT, $n^{\circ} 6,75 \mathrm{p}$.

9. PLUMELLE C. (1985), Renforcement d'un sol lâche par inclusion de micropieux. Revue Française de Géotechnique, n 30, pp. 47-57.

10. QUEYROI D., CHAPUT D., PILOT G. (1985), Amélioration des sols de fondation. Laboratoire Central des Ponts et Chaussées, note d'information technique, $55 \mathrm{p}$.

11. RANDOLPH M.F. (1983), Desing of piled raft foundations. In $\alpha$ Recent developments in laboratory and field tests and analysis of geotechnical problems », Bangkok, pp. 525-537.

12. RATHMAYER H. (1975), Piled embankment supported by single pile caps. Proceedings, Istanbul Conférence on Soil Mechanics and Foundation Engineering, $8 \mathrm{p}$.

13. REID W.M., BUCHANAN N.W. (1983), Bridge approach support piling. In * Piling and ground treatment s, Institution of civil Engineers, London, pp. $267-274$.

14. SOUTO E.B., PRADO N.T., CASTRO C.F.C. (1983), Iron-ore stockage on solt clays. Comptes rendus $7^{\circ}$ Congrès Panaméricain de Mécanique des sols et des Travaux de fondations, Vancouver, vol. II, pp. 499-506.

15. THORBURN S., LAIRD C.L, RANDOLPH M.F. (1983), Piling and ground treatment. Institution of civil Engineers, London, pp. 157-164.

16. TING W.H., TOH C.T., CHAN S.F. (1983), Pile supported fill. In * Recent developpements in laboratory and field tests and analysis of geotechnical problems », Bangkok, 1983, pp. 95-100 